TRANSACTIONS OF THE

AMERICAN MATHEMATICAL SOCIETY

Volume 359, Number 12, December 2007, Pages 6027-6055

S 0002-9947(07)04209-2

Article electronically published on June 13, 2007

\title{
QUOTIENTS OF STANDARD HILBERT MODULES
}

\author{
WILLIAM ARVESON
}

\begin{abstract}
We initiate a study of Hilbert modules over the polynomial algebra $\mathcal{A}=\mathbb{C}\left[z_{1}, \ldots, z_{d}\right]$ that are obtained by completing $\mathcal{A}$ with respect to an inner product having certain natural properties. A standard Hilbert module is a finite multiplicity version of one of these. Standard Hilbert modules occupy a position analogous to that of free modules of finite rank in commutative algebra, and their quotients by submodules give rise to universal solutions of nonlinear relations. Essentially all of the basic Hilbert modules that have received attention over the years are standard, including the Hilbert module of the $d$-shift, the Hardy and Bergman modules of the unit ball, modules associated with more general domains in $\mathbb{C}^{d}$, and those associated with projective algebraic varieties.

We address the general problem of determining when a quotient $H / M$ of an essentially normal standard Hilbert module $H$ is essentially normal. This problem has been resistant. Our main result is that it can be "linearized" in that the nonlinear relations defining the submodule $M$ can be reduced, appropriately, to linear relations through an iteration procedure, and we give a concrete description of linearized quotients.
\end{abstract}

\section{INTRODUCTION}

Let $T_{1}, \ldots, T_{d}$ be a commuting $d$-tuple of operators on a Hilbert space $H$. One can view $H$ as a module over the polynomial algebra $\mathcal{A}=\mathbb{C}\left[z_{1}, \ldots, z_{d}\right]$ in the natural way

$$
f \cdot \xi=f\left(T_{1}, \ldots, T_{d}\right) \xi, \quad f \in \mathcal{A}, \quad \xi \in H,
$$

and such an $H$ is called a Hilbert module of dimension $d$, or simply a Hilbert module when the dimension is understood. A Hilbert module is said to be essentially normal if the self-commutators $T_{k}^{*} T_{j}-T_{j} T_{k}^{*}$ of its ambient operators are all compact, and more specifically, p-essentially normal if the self-commutators belong to the Schatten class $\mathcal{L}^{p}, p$ being a number in the interval $[1, \infty]$, where $\mathcal{L}^{\infty}$ is interpreted as the $C^{*}$-algebra $\mathcal{K}$ of compact operators on $H$.

As in algebra, examples in multivariable operator theory are constructed most naturally through the formation of quotients, i.e., by passing from the simplest "free" Hilbert modules $H$ to their quotient Hilbert modules $H / M$, in which $M$ is the closed submodule of $H$ generated by the relations one seeks to satisfy. However, in the operator-theoretic context, properties such as essential normality, Fredholmness, and finiteness of the cohomology of the associated Koszul complex, do not

Received by the editors July 19, 2005 and, in revised form, November 5, 2005.

2000 Mathematics Subject Classification. Primary 46L07, 47A99.

The author was supported by NSF grant DMS-0100487.

(C)2007 American Mathematical Society Reverts to public domain 28 years from publication 
propagate naturally from $H$ to its submodules or quotients, with the result that it is unclear whether Hilbert modules with the sought-after properties exist.

For example, consider the problem of constructing operator solutions $X_{1}, \ldots, X_{d}$ to systems of algebraic equations of the form

$$
f_{k}\left(X_{1}, \ldots, X_{d}\right)=0, \quad k=1, \ldots, r,
$$

where the $f_{k}$ are homogeneous polynomials in $d$ variables and $X_{1}, \ldots, X_{d}$ are mutually commuting bounded operators on a Hilbert space $H$. We can make the point well enough with the following special case, in which one fixes a positive integer $n \geq 2$ and seeks a commuting triple $X, Y, Z \in \mathcal{B}(H)$ with the property

$$
X^{n}+Y^{n}=Z^{n} \text {. }
$$

Such a triple can be viewed as a "noncommutative curve". We say noncommutative in order to rule out variants of classical solutions such as those obtained by assembling a sequence of numerical solutions $\left(x_{k}, y_{k}, z_{k}\right) \in \mathbb{C}^{3}, k \geq 1$, of $x^{n}+y^{n}=z^{n}$ into diagonal operators acting on $\ell^{2}(\mathbb{N})$ such as

$$
X=\left(\begin{array}{cccc}
x_{1} & 0 & 0 & \cdots \\
0 & x_{2} & 0 & \cdots \\
0 & 0 & x_{3} & \cdots \\
\vdots & \vdots & \vdots &
\end{array}\right), Y=\left(\begin{array}{cccc}
y_{1} & 0 & 0 & \cdots \\
0 & y_{2} & 0 & \cdots \\
0 & 0 & y_{3} & \cdots \\
\vdots & \vdots & \vdots &
\end{array}\right), Z=\left(\begin{array}{cccc}
z_{1} & 0 & 0 & \cdots \\
0 & z_{2} & 0 & \cdots \\
0 & 0 & z_{3} & \cdots \\
\vdots & \vdots & \vdots &
\end{array}\right)
$$

While, in general, the operators $X, Y, Z$ of (1.2) must commute, the unital $C^{*}$ algebra $C^{*}(X, Y, Z)$ that they generate is typically noncommutative. We seek solution triples $X, Y, Z$ that are "universal" in an appropriate sense, that generate an irreducible $C^{*}$-algebra containing the $C^{*}$-algebra $\mathcal{K}$ of compact operators, and that are commutative modulo $\mathcal{K}$. The latter properties are summarized in an exact sequence of $C^{*}$-algebras

$$
0 \longrightarrow \mathcal{K} \longrightarrow C^{*}(X, Y, Z) \longrightarrow C(V) \longrightarrow 0,
$$

in which $V$ is a compact subset of $\left\{(x, y, z) \in \mathbb{C}^{3}: x^{n}+y^{n}=z^{n}\right\}$. The sequence (1.3) defines an extension of $\mathcal{K}$ by $C(V)$ in the sense of Brown-Douglas-Fillmore and, as shown in BDF77, it gives rise to an element of the $K$-homology of the space $V$. Of course, similar comments apply to the more general "noncommutative varieties" defined by operator solutions $X_{1}, \ldots, X_{d}$ of systems of equations of the form (1.1).

Following basic principles, one constructs solutions of algebraic equations such as (1.2) by passing from the polynomial algebra $\mathbb{C}[x, y, z]$ to its quotient by the ideal generated by $x^{n}+y^{n}-z^{n}$. The elements $\dot{x}, \dot{y}, \dot{z}$ obtained by projecting $x, y, z$ to the quotient are forced to satisfy $\dot{x}^{n}+\dot{y}^{n}=\dot{z}^{n}$, and there is an obvious sense in which this solution $\dot{x}, \dot{y}, \dot{z}$ is the universal one.

When one attempts to carry out this construction of solutions in the context of Hilbert space operators, one encounters an exasperating difficulty. To illustrate the point, let us complete the polynomial algebra $\mathbb{C}[x, y, z]$ in an appropriate inner product (for definiteness we choose the inner product associated with the 3-shift [Arv98]) giving rise to a Hilbert space $H^{2}(x, y, z)$. The operators $X, Y, Z$ on $H^{2}(x, y, z)$ defined by multiplication by the basic variables $x, y, z$ are bounded, and we may form the closed invariant subspace $M \subseteq H^{2}(x, y, z)$ generated by $x^{n}+y^{n}-z^{n}$ and its Hilbert space quotient $H=H^{2}(x, y, z) / M$. The operators $X, Y, Z$ promote naturally to operators $\dot{X}, \dot{Y}, \dot{Z}$ on the quotient $H$, the promoted operators satisfy (1.2), 
and straightforward computation shows that the $C^{*}$-algebra $C^{*}(\dot{X}, \dot{Y}, \dot{Z})$ is irreducible and contains $\mathcal{K}$. Thus, if one knew that all self-commutators $\dot{X}^{*} \dot{X}-\dot{X} \dot{X}^{*}$, $\dot{X}^{*} \dot{Y}-\dot{Y} \dot{X}^{*}, \ldots$, were compact, then $C^{*}(\dot{X}, \dot{Y}, \dot{Z})$ would be commutative modulo $\mathcal{K}$ and we would have an extension of the desired form

$$
0 \longrightarrow \mathcal{K} \longrightarrow C^{*}(\dot{X}, \dot{Y}, \dot{Z}) \longrightarrow C(V) \longrightarrow 0 .
$$

The difficulty is that it is unknown if $C^{*}(\dot{X}, \dot{Y}, \dot{Z})$ is commutative modulo $\mathcal{K}$ for any $n \geq 31$

The difficulty only grows in more general settings. For example, suppose that $M$ is a graded submodule of the finite multiplicity Hilbert module $H^{2}(x, y, z) \otimes \mathbb{C}^{r}$, and let $H$ be the quotient Hilbert module

$$
H=\frac{H^{2}(x, y, z) \otimes \mathbb{C}^{r}}{M} .
$$

This quotient $H$ no longer corresponds so simply to solutions of equations such as (1.2), but could represent a Hilbert space of sections of a vector bundle or sheaf over an appropriate algebraic set. Interpretations aside, one still has the basic operator-theoretic question as to whether the $C^{*}$-algebra $C^{*}(\dot{X}, \dot{Y}, \dot{Z})$ generated by the natural operators of $H$ is commutative modulo $\mathcal{K}$. Again, the answer is unknown in most cases of interest.

The purpose of this paper is to initiate the study of a broad context in which one can confront this issue, and which contains all of the important examples. We show that the general problem of proving essential normality of quotient modules defined by nonlinear relations can be reduced to the case in which the relations that define the quotient module are linear. Despite the explicit formulation of the linearized problem, it remains unsolved in general. Problems and conjectures are discussed in Section 9 .

Initially, we took up this program out of a desire to give a natural proof that the curvature invariant of Arv00, Arv02 is stable under compact perturbations and homotopy, by showing that pure finite-rank graded $d$-contractions satisfy the Fredholm property. In turn, that question led us to attempt to establish essential normality for certain quotient Hilbert modules (see Proposition 4.2 below). The preceding discussion shows that other fundamental issues of operator theory lead naturally to the same problem.

\section{Graded completions of $\mathbb{C}\left[z_{1}, \ldots, z_{d}\right]$}

We consider Hilbert modules $G$ obtained by completing the algebra of complex polynomials $\mathcal{A}=\mathbb{C}\left[z_{1}, \ldots, z_{d}\right]$ in an inner product with the property that the natural multiplication operators $Z_{1}, \ldots, Z_{d}$ associated with the generators $z_{1}, \ldots, z_{d}$ are bounded. One can increase multiplicity by forming the direct sum of $r<\infty$ copies $G \oplus \cdots \oplus G=G \otimes \mathbb{C}^{r}$ of such a module $G$, which is a Hilbert module whose natural operators are multiplicity $r$ versions of the original $Z_{k}$. It is convenient to abuse notation by also writing these finite multiplicity multiplication operators as $Z_{1}, \cdots, Z_{d}$.

In this section we single out a class of inner products on the algebra $\mathcal{A}$ whose completions are Hilbert modules, the finite multiplicity versions of which form effective building blocks for multivariable operator theory in Hilbert spaces. We call them

\footnotetext{
${ }^{1}$ Added in proof: A recent revision of [GW05] asserts that in these examples, $\dot{X}, \dot{Y}, \dot{Z}$ is $p$-essentially normal for every $p>3$. The corresponding problem for systems (1.1) remains open.
} 
standard Hilbert modules. This is a very broad class of Hilbert modules that differs in several ways from classes that have been previously studied [MV93, [DMV00, DM03. For example, standard Hilbert modules are not necessarily subnormal, nor are they necessarily associated with a reproducing kernel.

Indeed, standard Hilbert modules occupy a position analogous to that of free modules in the algebraic theory of finitely generated modules over $\mathcal{A}$; they are basically the Hilbert modules that have the same cohomology as free modules in the algebraic theory (see Remark 5.3). However, while there is only one algebraic free module of rank one, namely $\mathcal{A}$ itself, there are many inequivalent standard Hilbert modules of rank one. That class of Hilbert modules includes all of the basic examples that have been studied in recent years, including the space $H^{2}$ of the $d$-shift, the Hardy space of the unit sphere in $\mathbb{C}^{d}$, the Bergman space of the unit ball, as well as Hilbert modules associated with other domains in $\mathbb{C}^{d}$ and projective algebraic varieties.

Remark 2.1 (Graded inner products on $\mathcal{A}$ ). There is a natural action $\Gamma$ of the circle group on $\mathcal{A}$ defined by

$$
\Gamma(\lambda) f\left(z_{1}, \ldots, z_{d}\right)=f\left(\lambda z_{1}, \ldots, \lambda z_{d}\right), \quad f \in \mathcal{A}, \quad \lambda \in \mathbb{T} .
$$

We write $\mathcal{A}_{n}$ for the linear space of homogeneous polynomials of degree $n$,

$$
\mathcal{A}_{n}=\left\{f \in \mathcal{A}: \Gamma(\lambda) f=\lambda^{n} f, \quad \lambda \in \mathbb{T}\right\}, \quad n=0,1,2, \ldots,
$$

and the polynomial algebra decomposes into an algebraic direct sum of homogeneous subspaces

$$
\mathcal{A}=\mathbb{C} \dot{+} \mathcal{A}_{1} \dot{+} \mathcal{A}_{2} \dot{+} \cdots \text {. }
$$

An inner product $\langle\cdot, \cdot\rangle$ on $\mathcal{A}$ is invariant under the action of $\Gamma$, i.e.,

$$
\langle\Gamma(\lambda) f, \Gamma(\lambda) g\rangle=\langle f, g\rangle, \quad f, g \in \mathcal{A}, \quad \lambda \in \mathbb{T},
$$

iff the homogeneous spaces are mutually orthogonal: $\left\langle\mathcal{A}_{m}, \mathcal{A}_{n}\right\rangle=\{0\}$ for $m \neq n$. Such inner products are called graded. If, for each $k=1, \ldots, d$, the multiplication operator $Z_{k}: f \mapsto z_{k} f$ is bounded relative to the norm $\|f\|=\langle f, f\rangle^{1 / 2}$, then the completion of $\mathcal{A}$ in this inner product is a Hilbert module over $\mathcal{A}$.

Definition 2.2. A graded completion of $\mathcal{A}$ is a Hilbert module $G$ obtained by completing $\mathcal{A}$ in a graded inner product with the additional property that the linear space $Z_{1} G+\cdots+Z_{d} G$ is closed.

Examples of graded completions include the Bergman and Hardy modules of the ball $\left\{z \in \mathbb{C}^{d}:\|z\|<1\right\}$, the space $H^{2}$ of the $d$-shift, the Bergman and Hardy modules of $d$-dimensional polydisks, and in fact most Bergman modules of more general domains in $\mathbb{C}^{d}$ that admit circular symmetry.

Remark 2.3 (Normalization of coordinates). In every graded completion $G$ of $\mathcal{A}=$ $\mathbb{C}\left[z_{1}, \ldots, z_{d}\right]$, we can replace the indicated basis $z_{1}, \ldots, z_{d}$ for $\mathcal{A}_{1}$ with another basis $\tilde{z}_{1}, \ldots, \tilde{z}_{d}$, if necessary, to achieve the normalization $\left\langle\tilde{z}_{i}, \tilde{z}_{j}\right\rangle=\delta_{i j}$. While this has the effect of changing the original set of multiplication operators $Z_{1}, \ldots, Z_{d}$ into another linear basis $\tilde{Z}_{1}, \ldots, \tilde{Z}_{d}$ for the operator space they span, it does not significantly affect properties of the Hilbert module $G$. For example, one shows easily that if the self-commutators of the original operators $Z_{j}^{*} Z_{k}-Z_{k} Z_{j}^{*}$ belong to $\mathcal{L}^{p}$, then so do the self-commutators $\tilde{Z}_{j}^{*} \tilde{Z}_{k}-\tilde{Z}_{k} \tilde{Z}_{j}^{*}$. Thus, we can assume throughout that for any graded completion $G$, the coordinates $z_{1}, \ldots, z_{d}$ are an orthonormal subset of $G$. 
For every graded completion $G$, the representation $\Gamma$ extends naturally to a strongly continuous unitary representation of $\mathbb{T}$ on $G$ (also written $\Gamma$ ) whose spectral subspaces

$$
G_{n}=\left\{\xi \in G: \Gamma(\lambda) \xi=\lambda^{n} \xi\right\}, \quad n \in \mathbb{Z}
$$

vanish for negative $n$, satisfy $G_{n}=\mathcal{A}_{n}$ for $n \geq 0$, and one has

$$
G=G_{0} \oplus G_{1} \oplus G_{2} \oplus \cdots .
$$

The space $Z_{1} G+\cdots+Z_{d} G$ is the orthocomplement of the one-dimensional space of constants $\mathbb{C} \cdot 1$. $\Gamma$ is called the gauge group of $G$; it relates to the ambient operators by way of $\Gamma(\lambda) Z_{k} \Gamma(\lambda)^{*}=\lambda Z_{k}, k=1, \ldots, d, \lambda \in \mathbb{T}$.

Remark 2.4 (Irreducibility). A submodule $M \subseteq H$ of a Hilbert module $H$ is said to be reducing if its orthocomplement $M^{\perp}$ is also a submodule. In this case we also refer to $M$ as a summand since it gives rise to a decomposition $H=M \oplus N$ of $H$ into a direct sum of Hilbert modules. $H$ is said to be irreducible if it has no nontrivial summands. Equivalently, $H$ is irreducible iff the $*$-algebra generated by the ambient operators $Z_{1}, \ldots, Z_{d}$ of $H$ has commutant $\mathbb{C} \cdot \mathbf{1}_{H}$.

$C^{*}\left(Z_{1}, \ldots, Z_{d}\right)$ will denote the unital $C^{*}$-algebra generated by the coordinate operators $Z_{1}, \ldots, Z_{d}$ of a Hilbert module $H$, and $\mathcal{K}$ will denote the $C^{*}$-algebra of all compact operators on $H$.

Proposition 2.5. Every graded completion of $\mathcal{A}$ is irreducible, and, in fact, $C^{*}\left(Z_{1}, \ldots, Z_{d}\right)$ contains $\mathcal{K}$.

Proof. Let $G$ be a graded completion and let $P \in \mathcal{B}(G)$ be a projection that commutes with the coordinate operators $Z_{1}, \ldots, Z_{d}$ of $G$. Choose a unit vector $v \in G_{0}$, so that $G=[\mathcal{A} v]$. It follows that $G_{0}=[v]$ is the orthocomplement of the closed subspace

$$
Z_{1} G+\cdots+Z_{d} G .
$$

Since $P$ commutes with each $Z_{k}$ it commutes with $Z_{1} Z_{1}^{*}+\cdots+Z_{d} Z_{d}^{*}$, and therefore with the projection onto the range of the latter operator, namely the subspace $Z_{1} G+\cdots+Z_{d} G$. Hence $P$ commutes with the rank-one projection $v \otimes \bar{v}$, so we must have either $P v=v$ or $P v=0$.

If $P v=v$, then $P$ must restrict to the identity operator on the closed submodule $[\mathcal{A} v]=G$ generated by $v$; hence $P=1$. Similarly, $P v=0$ implies $P=0$; we conclude that $C^{*}\left(Z_{1}, \ldots, Z_{d}\right)$ is irreducible.

Thus, to show that $C^{*}\left(Z_{1}, \ldots, Z_{d}\right)$ contains all compact operators, it suffices to observe that $C^{*}\left(Z_{1}, \ldots, Z_{d}\right)$ contains a rank-one projection, namely $\mathbf{1}-R$, where $R$ is the projection onto $Z_{1} H+\cdots+Z_{d} H$ (see Remark 2.8). Since $Z_{1} G+\cdots+Z_{d} G$ is closed it coincides with the range of $Z_{1} Z_{1}^{*}+\cdots+Z_{d} Z_{d}^{*}$, so that 0 is an isolated point in the spectrum of the latter operator. A familiar argument with the functional calculus exhibits the projection $R$ as a continuous function of $Z_{1} Z_{1}^{*}+\cdots+Z_{d} Z_{d}^{*}$; hence $R \in C^{*}\left(Z_{1}, \ldots, Z_{d}\right)$.

Remark 2.6 (Number operator). The number operator of a graded completion $G$ is defined as the self-adjoint generator $N$ of the gauge group

$$
\Gamma\left(e^{i t}\right)=e^{i t N}, \quad t \in \mathbb{R} .
$$

The number operator is self-adjoint, has integer spectrum $\{0,1,2, \ldots\}$ and its minimal spectral projections are the projections onto the homogeneous spaces $G_{n}=\mathcal{A}_{n}$, 
$n=0,1,2, \ldots$. Since the dimensions of the spaces $\mathcal{A}_{n}$ do not depend on the inner product chosen, any two graded completions of $\mathcal{A}$ have unitarily equivalent number operators. It was shown in the appendix of Arv98 that the number operator satisfies

$$
(N+\mathbf{1})^{-1} \in \mathcal{L}^{p} \Longleftrightarrow p>d .
$$

Remark 2.7 (Graded Hilbert modules, Gauge groups). More general graded Hilbert modules $H$ can be defined in two equivalent ways. One specifies either a $\mathbb{Z}$-grading for $H$,

$$
H=\cdots \oplus H_{-1} \oplus H_{0} \oplus H_{1} \oplus \cdots,
$$

in which $Z_{k} H_{n} \subseteq H_{n+1}$, for all $1 \leq k \leq d, n \in \mathbb{Z}$, or one specifies a gauge group, a strongly continuous unitary representation $\Gamma$ of the circle group $\mathbb{T}$ on $H$, such that

$$
\Gamma(\lambda) Z_{k} \Gamma(\lambda)^{*}=\lambda Z_{k} \quad 1 \leq k \leq d, \quad \lambda \in \mathbb{T} .
$$

One passes back and forth via the associations $H_{n}=\left\{\xi \in H: \Gamma(\lambda) \xi=\lambda^{n} \xi\right\}$ and $\Gamma(\lambda)=\sum_{n} \lambda^{n} E_{n}, E_{n}$ being the projection on $H_{n}, n \in \mathbb{Z}$. In a graded Hilbert module with projections $E_{n}$ as above, one has the commutation relations $Z_{k} E_{n}=$ $E_{n+1} Z_{k}, 1 \leq k \leq d, n \in \mathbb{Z}$.

The graded Hilbert modules that we encounter in this paper will all have nonnegative spectrum in the sense that $H_{n}=\{0\}$ for all $n<0$.

Remark 2.8 (Characterization of graded completions). Graded completions can be characterized abstractly as graded Hilbert modules $H=H_{0} \oplus H_{1} \oplus \cdots$ satisfying

A. $H_{0}=[v]$ is one-dimensional,

B. $Z_{1} H+\cdots+Z_{d} H$ is closed,

for which the map

$$
f \in \mathcal{A} \mapsto f\left(Z_{1}, \ldots, Z_{d}\right) v \in H
$$

is injective with dense range. It follows that the homogeneous subspaces of such a Hilbert module satisfy

$$
H_{n+1}=Z_{1} H_{n}+\cdots+Z_{d} H_{n}, \quad n=0,1,2, \ldots
$$

\section{Standard Hilbert modules and their quotients}

One can increase the multiplicity of a graded completion $G$ to obtain a somewhat more general graded Hilbert module. In more detail, let $E$ be a finite-dimensional Hilbert space and let $S=G \otimes E$ be the Hilbert module defined by

$$
f(\xi \otimes \zeta)=(f \cdot \xi) \otimes \zeta, \quad f \in \mathcal{A}, \quad \xi \in G, \quad \zeta \in E .
$$

Thus, the coordinate operators $\tilde{Z}_{k}$ of $S$ are related to the operators $Z_{k}$ of $G$ by $\tilde{Z}_{k}=Z_{k} \otimes \mathbf{1}_{E}$. It is convenient to ease notation by writing $Z_{k}$ for $Z_{k} \otimes \mathbf{1}_{E}$, and we usually do so. We occasionally write $r \cdot G$ for $G \otimes \mathbb{C}^{r}$.

Definition 3.1. A standard Hilbert module is a finite-multiplicity version $S=G \otimes E$ of a graded completion $G$.

When we want to call attention to the underlying graded completion, we say that a standard Hilbert module $S$ is based on $G$ when it has the above form $S=G \otimes E$. Obviously, the direct sum of two standard Hilbert modules based on $G$ is a standard Hilbert module based on $G$. On the other hand, it is important to keep in mind that direct sums are not allowed across the category of graded completions. Indeed, if $G_{1}$ and $G_{2}$ are two graded completions of $\mathcal{A}$ that are associated with different 
inner products, then while the direct sum $G_{1} \oplus G_{2}$ is certainly a graded Hilbert module, it need not be a standard Hilbert module (based on any $G$ ), and it may fail to have the favorable properties of standard Hilbert modules.

Standard Hilbert modules carry an obvious grading $H=H_{0} \oplus H_{1} \oplus \cdots$. For example, the gauge group of $G \otimes E$ is $\Gamma(\lambda)=\Gamma_{0}(\lambda) \otimes \mathbf{1}_{E}, \lambda \in \mathbb{T}$, $\Gamma_{0}$ being the gauge group of $G$. Note too that for any standard Hilbert module $S$, the space

$$
Z_{1} S+\cdots+Z_{d} S=\left(Z_{1} G+\cdots+Z_{d} G\right) \otimes E
$$

is closed, and we have

$$
S_{n+1}=Z_{1} S_{n}+\cdots+Z_{d} S_{n}, \quad n=0,1,2, \cdots
$$

Standard Hilbert modules are of degree 0 in the sense of the following general definition.

Definition 3.2 (Degree of a graded module). Let $H=H_{0} \oplus H_{1} \oplus H_{2} \oplus \cdots$ be a graded Hilbert module. The degree of $H$ is the smallest integer $n \geq 0$ such that

$$
H_{k+1}=Z_{1} H_{k}+\cdots+Z_{d} H_{k}, \quad k \geq n .
$$

If there is no such $n \in \mathbb{Z}_{+}$, then the degree of $H$ is defined as $\infty$.

Hilbert's Basis Theorem implies that every graded submodule of a finitely generated graded module over the polynomial algebra $\mathcal{A}$ has a finite number of homogeneous generators. It is a straightforward exercise to apply that fact to deduce the following:

Proposition 3.3. For every graded submodule $M=M_{0} \oplus M_{1} \oplus \cdots$ of a standard Hilbert module $S=G \otimes E$ there is an $n=0,1,2, \ldots$ such that

$$
M_{k+1}=Z_{1} M_{k}+\cdots+Z_{d} M_{k}, \quad k \geq n .
$$

We conclude that every graded submodule of a standard Hilbert module is of finite nonnegative degree.

Remark 3.4 (Degree of submodules). A direct application of Proposition 2.5] shows that the reducing submodules of a standard Hilbert module $G \otimes E$ are the submodules $G \otimes F$, where $F$ is a linear subspace of $E$. It follows that a graded submodule $M=M_{0} \oplus M_{1} \oplus \cdots$ of a standard Hilbert module is a reducing submodule iff it is of degree 0 . More generally, every graded submodule $M$ of $G \otimes E$ of degree $n \geq 1$ admits a representation

$$
M=M_{0} \oplus M_{1} \oplus \cdots \oplus M_{n-1} \oplus\left[\mathcal{A} M_{n}\right],
$$

where $M_{k} \subseteq \mathcal{A}_{k} \otimes E$ is a linear space of homogeneous vector polynomials.

Let $S=G \otimes E$ be a standard Hilbert module based on a graded completion $G$. We are primarily interested in properties of the quotient modules $H=S / M$, where $M$ is a graded submodule of $S$. Such quotient modules carry a natural grading $H=H_{0} \oplus H_{1} \oplus \cdots$, in which $H_{k}=S_{k} / M_{k}, k=0,1,2, \ldots$ A quotient module $H=(G \otimes E) / M$ of this form is called a $G$-quotient. If the submodule $M$ is of degree $n=0,1,2, \ldots$, we often refer to the quotient as a $G(n)$-quotient.

Remark 3.5 (Ambiguity in the notion of $G(n)$-quotients). Several things must be kept in mind when dealing with quotients of standard Hilbert modules. 
1. Consider first the case in which $G$ is the module $H^{2}$ of the $d$-shift. The dilation theory of $d$-contractions implies that there is an intrinsic characterization of $H^{2}$ quotients up to unitary equivalence: A graded Hilbert module $H=H_{0} \oplus H_{1} \oplus \cdots$ is unitarily equivalent to an $H^{2}$-quotient iff its coordinate operators $T_{1}, \ldots, T_{d}$ define a pure finite rank $d$-contraction (we oversimplify slightly in order to make the essential point; see Arv98] and Arv00] for more details). On the other hand, there is no known characterization of $H^{2}$-quotients up to isomorphism, or even $p$ isomorphism for $p>d$ ( $p$-morphisms are introduced Section 4). For more general graded completions $G$ in place of $H^{2}$, very little is known about the characterization of $G$-quotients - even up to unitary equivalence.

2. In more explicit terms, given that one knows somehow that a graded Hilbert module $H$ is isomorphic to a $G$-quotient for some graded completion $G$, one does not know how to use the structure of $H$ to obtain information about $G$. One does not even know if the structure of some particular $G$-quotient $H$ determines $G$ up to isomorphism; though we lack a specific example it seems likely that a $G$-quotient can be isomorphic to a $G^{\prime}$-quotient when $G$ and $G^{\prime}$ are graded completions that are not isomorphic.

3. Even when one works within the category of $G$-quotients for a fixed graded completion $G$, there is still ambiguity in the notion of a $G(n)$-quotient, since $n$ is not uniquely determined by the structure of a $G(n)$-quotient. Indeed, in the following sections we exploit this ambiguity by showing that, up to finite-dimensional perturbations, a $G(n)$-quotient for $n \geq 2$ can always be realized up to isomorphism as a $G(1)$-quotient, namely a quotient of the form $(G \otimes E) / M$ where $M \subseteq G \otimes E$ is a graded submodule of degree one.

\section{4. $p$-ESSENTIAL NORMALITY, $p$-MORPHISMS}

Let $H$ and $K$ be Hilbert spaces. For $p \in[1, \infty]$, we write $\mathcal{L}^{p}(H, K)$, or more simply $\mathcal{L}^{p}$, for the Schatten-von Neumann class of all operators $A \in \mathcal{B}(H, K)$ whose modulus $|T|=\sqrt{T^{*} T}$ satisfies trace $|T|^{p}<\infty$ when $p<\infty, \mathcal{L}^{\infty}$ being interpreted as the space of compact operators $\mathcal{K}(H, K)$.

Definition 4.1. Let $p \in[1, \infty]$ and let $H$ be a Hilbert module with coordinate operators $A_{1}, \ldots, A_{d}$. $H$ is said to be $p$-essentially normal if the self-commutators $A_{j} A_{k}^{*}-A_{k}^{*} A_{j}$ belong to $\mathcal{L}^{p}$ for all $1 \leq j, k \leq d$.

We refer to $\infty$-essentially normal Hilbert modules with the shorter term essentially normal. We will make repeated use of the following general result which asserts, roughly, that submodules and quotients of essentially normal Hilbert modules are either very good or very bad. Notice that neither the statement nor proof of Proposition 4.2 provides information about how one might establish the favorable properties.

Proposition 4.2. Let $p \in[1, \infty]$ and let $H$ be a p-essentially normal Hilbert module such that $T_{1} H+\cdots+T_{d} H$ is a closed subspace of finite codimension in $H$. For every submodule $M \subseteq H$ such that $\{0\} \neq M \neq H$ with projection $P=P_{M}$, the following are equivalent:

(i) $M$ is p-essentially normal.

(ii) $H / M$ is p-essentially normal.

(iii) Each commutator $\left[P, T_{1}\right], \ldots,\left[P, T_{d}\right]$, belongs to $\mathcal{L}^{2 p}$.

(iv) $\left[P, T_{k}\right]^{*}\left[P, T_{j}\right] \in \mathcal{L}^{p}$ for $1 \leq j, k \leq d$. 
(v) $\left[P, T_{j}\right]\left[P, T_{k}\right]^{*} \in \mathcal{L}^{p}$ for $1 \leq j, k \leq d$.

(vi) $M$ is p-essentially normal and $T_{1} M+\cdots+T_{d} M$ is a closed subspace of finite codimension in $M$.

(vii) $H / M$ satisfies the conditions of (vi).

Proof. While the proof is a straightforward variation of the proof of Proposition 4.1 of [Arv04, we present the details for completeness. Properties (vi) and (vii) obviously imply (i) and (ii), respectively.

(iii) $\Longleftrightarrow$ (iv) $\Longleftrightarrow$ (v): Consider the row operator whose entries are the commutators $C=\left(\left[P, T_{1}\right],\left[P, T_{2}\right], \ldots,\left[P, T_{d}\right]\right) . C^{*} C$ is the $d \times d$ matrix $\left(A_{i j}\right)$ with entries $A_{i j}=\left[P, T_{i}\right]^{*}\left[P, T_{j}\right]$. After noting that $C^{*} C \in \mathcal{L}^{p}$ iff $C \in \mathcal{L}^{2 p}$, the equivalence (iii) $\Longleftrightarrow$ (iv) follows. The proof of (iii) $\Longleftrightarrow$ (v) is similar.

(i) $\Longleftrightarrow(\mathrm{v})$ : Letting $B_{j}$ be the restriction of $T_{j}$ to $M$, we claim

$$
\left[B_{j}, B_{k}^{*}\right] P=-\left[P, T_{j}\right]\left[P, T_{k}\right]^{*}+P\left[T_{j}, T_{k}^{*}\right] P .
$$

Indeed, for each $1 \leq j, k \leq d$ we can write

$$
\begin{aligned}
{\left[B_{j}, B_{k}^{*}\right] P } & =T_{j} P T_{k}^{*} P-P T_{k}^{*} T_{j} P=T_{j} P T_{k}^{*} P-P T_{j} T_{k}^{*} P+P\left[T_{j}, T_{k}^{*}\right] P \\
& =-P T_{j} P^{\perp} T_{k}^{*} P+P\left[T_{j}, T_{k}^{*}\right] P .
\end{aligned}
$$

Since $P T_{j} P^{\perp}=P T_{j}-T_{j} P$, we have $P T_{j} P^{\perp} T_{k}^{*} P=\left[P, T_{j}\right]\left[P, T_{k}\right]^{*}$, and (4.1) follows. Since $H$ is $p$-essentially normal we have $\left[T_{j}, T_{k}^{*}\right] \in \mathcal{L}^{p}$, and at this point the equivalence (i) $\Longleftrightarrow$ (iv) follows from (4.1).

(i) $\Longleftrightarrow$ (iv): Letting $C_{j}$ be the compression of $T_{j}$ to $M^{\perp}$, we claim

$$
\left[C_{j}, C_{k}^{*}\right] P^{\perp}=\left[P, T_{k}\right]^{*}\left[P, T_{j}\right]+P^{\perp}\left[T_{j}, T_{k}^{*}\right] P^{\perp},
$$

$P^{\perp}$ denoting the projection on $M^{\perp}$. Noting that $P^{\perp} T_{j} P^{\perp}=P^{\perp} T_{j}$, one has

$$
\begin{aligned}
{\left[C_{j}, C_{k}^{*}\right] P^{\perp} } & =P^{\perp} T_{j} T_{k}^{*} P^{\perp}-P^{\perp} T_{k}^{*} P^{\perp} T_{j} P^{\perp} \\
& =P^{\perp} T_{k}^{*} T_{j} P^{\perp}-P^{\perp} T_{k}^{*} P^{\perp} T_{j} P^{\perp}+P^{\perp}\left[T_{j}, T_{k}^{*}\right] P^{\perp} \\
& =P^{\perp} T_{k}^{*} P T_{j} P^{\perp}+P^{\perp}\left[T_{j}, T_{k}^{*}\right] P^{\perp} .
\end{aligned}
$$

(4.2) follows after one notes that $P^{\perp} T_{k}^{*} P T_{j} P^{\perp}=\left[P, T_{k}\right]^{*}\left[P, T_{j}\right]$. Again, we have $\left[T_{j}, T_{k}^{*}\right] \in \mathcal{L}^{p}$ by hypothesis, and the equivalence (i) $\Longleftrightarrow$ (iv) follows from formula (4.2).

(i) $\Longrightarrow$ (iv): Assuming (i), we have to show that $T_{1} M+\cdots+T_{d} M$ is closed of finite codimension. This will follow if we show that the restrictions $A_{k}=T_{k} \Upsilon_{M}$ have the property that $A_{1} A_{1}^{*}+\cdots+A_{d} A_{d}^{*}$ is a Fredholm operator.

For that, we show that this sum has the form $X+K$ where $X$ is a positive invertible operator and $K$ is compact. Indeed, the hypotheses on $H$ imply that $T_{1}^{*} T_{1}+\cdots+T_{d}^{*} T_{d}$ is a positive Fredholm operator, which therefore has the form $X_{0}+F$ where $X_{0}$ is a positive invertible operator and $F$ is a finite rank operator. Hence the compression of $X_{0}+F$ to $M$ has the form $X+K$ where $X=P_{M} X_{0} \Upsilon_{M}$ is a positive invertible operator on $M$ and $K$ is the finite rank compression of $F$ to $M$. Using $P_{M} T_{k} \Upsilon_{M}=T_{k} \uparrow_{M}$, we have

$$
A_{1}^{*} A_{1}+\cdots+A_{d}^{*} A_{d}=P_{M}\left(T_{1}^{*} T_{1}+\cdots+T_{d}^{*} T_{k}\right)\left\lceil_{M}=P_{M}\left(X_{0}+F\right)\left\lceil_{M}=X+K ;\right.\right.
$$

hence $A_{1}^{*} A_{1}+\cdots+A_{d}^{*} A_{d}$ is Fredholm. Since the self-commutators $\left[A_{k}^{*}, A_{k}\right]$ are compact by (i), it follows that $A_{1} A_{1}^{*}+\cdots+A_{d} A_{d}^{*}$ is a compact perturbation of $A_{1}^{*} A_{1}+\cdots+A_{d}^{*} A_{d}$, and is therefore Fredholm. 
(ii) $\Longrightarrow(\mathrm{v})$ : The argument is similar to the one above. One notes that the compressions $B_{k}=P_{M}^{\perp} T_{k} \uparrow_{M^{\perp}}$ of the coordinate operators to $M^{\perp}$ satisfy

$$
P_{M}^{\perp}\left(T_{1} T_{1}^{*}+\cdots+T_{d} T_{d}^{*}\right)\left\lceil_{M^{\perp}}=B_{1} B_{1}^{*}+\cdots+B_{d} B_{d}^{*}\right.
$$

and uses the fact that $T_{1} T_{1}^{*}+\cdots+T_{d} T_{d}^{*}$ is a positive Fredholm operator.

Obviously, the notion of $p$-essential normality is invariant under unitary equivalence of Hilbert modules. On the other hand, essential normality may not be preserved under more general isomorphisms of Hilbert modules, even under graded isomorphisms of graded Hilbert modules. Some elementary examples are described in Remark 4.7 below. Similarly, submodules and quotients of essentially normal Hilbert modules need not be essentially normal in general (some ungraded examples are exhibited in [GS05]). The purpose of this section is to establish general conditions under which $p$-essential normality does propagate to submodules, quotients, and to their images under homomorphisms and isomorphisms.

Definition 4.3 ( $p$-morphism). Let $H, K$ be Hilbert modules with respective operator $d$-tuples $A_{1}, \ldots, A_{d}, B_{1}, \ldots, B_{d}$, and let $p \in[1, \infty]$. By a $p$-morphism from $H$ to $K$ we mean a homomorphism $L \in \operatorname{hom}(H, K)$ with the additional property $L^{*} B_{k}-A_{k} L^{*} \in \mathcal{L}^{p}$ (or equivalently, $L A_{k}^{*}-B_{k}^{*} L \in \mathcal{L}^{p}$ ), $k=1, \ldots, d$. A p-isomorphism is an isomorphism of Hilbert modules that is also a $p$-morphism.

One checks readily that the inverse of a $p$-isomorphism $L: H \rightarrow K$ is a $p$ isomorphism $L^{-1}: K \rightarrow H$. The following result provides a context in which $p$ morphisms of submodules and quotient modules arise naturally, and it implies that $p$-essential normality of quotient modules propagates as desired under appropriate conditions.

Theorem 4.4. Fix $p \in[1, \infty]$. Let $H$ and $K$ be $p$-essentially normal Hilbert modules, let $L: H \rightarrow K$ be a $2 p$-morphism, and let $M \subseteq H$ be a submodule such that $L(M)$ is closed. If $M$ is p-essentially normal then:

(i) $L(M)$ and $M \cap \operatorname{ker} L$ are $p$-essentially normal submodules of $K$ and $M$, respectively.

(ii) The restriction of $L$ to $M$ defines a $2 p$-morphism in $\operatorname{hom}(M, L(M))$ and in $\operatorname{hom}(M, K)$.

(iii) The quotients $H / M$ and $K / L(M)$ are p-essentially normal, and the promoted map $\dot{L} \in \operatorname{hom}(H / M, K / L(M))$ is a $2 p$-morphism.

Proof. (i): Let $X_{1}, \ldots, X_{d}$ and $Y_{1}, \ldots, Y_{d}$ be the coordinate operators of $H$ and $K$ respectively, and consider the operator $B=L P_{M} L^{*} \in \mathcal{B}(K)$. Note that the commutators $\left[B, Y_{k}\right]$ must belong to $\mathcal{L}^{2 p}, k=1, \ldots, d$. Indeed, for each $k$ we have $Y_{k} L=L X_{k}, X_{k} L^{*}-L^{*} Y_{k} \in \mathcal{L}^{2 p}$ because $L$ is a $2 p$-morphism, and Proposition 4.2 implies that $X_{k} P_{M}-P_{M} X_{k} \in \mathcal{L}^{2 p}$ because $M$ is $p$-essentially normal. It follows that

$$
Y_{k} B-B Y_{k}=L\left(X_{k} P_{M}-P_{M} X_{k}\right) L^{*}+L P_{M}\left(X_{k} L^{*}-L^{*} Y_{k}\right) \in \mathcal{L}^{2 p} .
$$

Note too that, since $N=L(M)$ is closed, $B$ is a positive operator with closed range $N$; hence either $B$ is invertible or 0 is an isolated point in $\sigma(B)$. In either case, there is a positive distance $\epsilon>0$ between 0 and the remaining part $\sigma(B) \cap(0, \infty)$ of the spectrum of $B$.

Let $\Gamma$ be the counter-clockwise oriented curve consisting of a rectangle with corners $(\epsilon / 2, \epsilon / 2),(\epsilon / 2,-\epsilon / 2),(\|B\|+\epsilon / 2,-\epsilon / 2),(\|B\|+\epsilon / 2, \epsilon / 2)$. $\Gamma$ winds once 
around every point of $\sigma(B) \cap(0, \infty)$ and has 0 in its exterior, so the projection $P_{N}$ can be expressed as an operator-valued Riemann integral

$$
P_{N}=\frac{1}{2 \pi i} \int_{\Gamma} R_{\lambda} d \lambda
$$

$R_{\lambda}$ denoting the resolvent $R_{\lambda}=(\lambda-B)^{-1}$. Perhaps it is appropriate to point out that, while in general the right side of the displayed formula merely defines an idempotent with the same range as $B$, in this case the integral is a normal operator since the $R_{\lambda}$ are commuting normal operators, and a normal idempotent must be a self-adjoint projection. Hence it is $P_{N}$.

It follows that for any bounded derivation of $\mathcal{B}(K)$ of the form $D(T)=[Y, T]$, with $Y \in \mathcal{B}(K)$, we have

$$
D\left(P_{N}\right)=\frac{1}{2 \pi i} \int_{\Gamma} D\left(R_{\lambda}\right) d \lambda
$$

Notice next that

$$
D\left(R_{\lambda}\right)=R_{\lambda} D(B) R_{\lambda}, \quad \lambda \in \Gamma .
$$

Indeed, using $D(S) T+S D(T)=D(S T)$ we have

$$
D\left(R_{\lambda}\right)(\lambda-B)-R_{\lambda} D(B)=D\left(R_{\lambda}\right)(\lambda-B)+R_{\lambda} D(\lambda-B)=D(\mathbf{1})=0,
$$

and (4.4) follows after multiplying on the right by $(\lambda-B)^{-1}$.

Now take $Y=Y_{k}, k=1, \ldots, d$. Using (4.3) and (4.4), we obtain the formula

$$
\left[Y_{k}, P_{N}\right]=\frac{1}{2 \pi i} \int_{\Gamma} R_{\lambda}\left[Y_{k}, B\right] R_{\lambda} d \lambda .
$$

Since $\left[Y_{k}, B\right] \in \mathcal{L}^{2 p}$ and $\lambda \mapsto R_{\lambda}$ is continuous in the operator norm, it follows that $\lambda \in \Gamma \mapsto R_{\lambda}\left[Y_{k}, B\right] R_{\lambda}$ is a continuous function from $\Gamma$ into the Banach space $\mathcal{L}^{2 p}$. Hence (4.5) expresses $\left[Y_{k}, P_{N}\right]$ as a Riemann integral of a continuous $\mathcal{L}^{2 p}$-valued function, and this implies that $\left[Y_{k}, P_{N}\right] \in \mathcal{L}^{2 p}$.

Finally, since $N$ is a submodule of the $p$-essentially normal Hilbert module $K$, we may conclude from Proposition 4.2 that $N$ is a $p$-essentially normal submodule of $K$.

Let $L_{0}$ be the restriction of $L$ to $M$. To see that $\operatorname{ker} L_{0}=M \cap \operatorname{ker} L$ is a $p$ essentially normal submodule of $H$, consider the adjoint $L_{0}^{*}=P_{M} L^{*}$ of $L_{0}$ as an operator in $B(K, M)$. The range of $L_{0}^{*}$ is the closed subspace $M \ominus(M \cap \operatorname{ker} L)=M \ominus$ ker $L_{0}$. If we use the respective $d$-tuples $Y_{1}^{*}, \ldots, Y_{d}^{*}$ and $\left.P_{M} X_{1}^{*} \uparrow_{M}, \ldots, P_{M} X_{d}^{*}\right\rceil_{M}$ to make $K$ and $M$ into $p$-essentially normal Hilbert modules over $\mathcal{A}$, then by Proposition 4.2, $L_{0}^{*}$ becomes a $2 p$-morphism in $\operatorname{hom}(K, M)$. Thus the argument given above implies that the projection on $L_{0}^{*}(K)$ commutes modulo $\mathcal{L}^{2 p}$ with $P_{M} X_{1}^{*} \uparrow_{M}, \ldots, P_{M} X_{d}^{*} \uparrow_{M}$, and hence the projection on $M \cap \operatorname{ker} L$ commutes modulo $\mathcal{L}^{2 p}$ with $X_{1} \uparrow_{M}, \ldots, X_{d} \uparrow_{M}$. Another application of Proposition 4.2 shows that $M \cap \operatorname{ker} L$ is a $p$-essentially normal Hilbert module with respect to the action of $X_{1} \uparrow_{M}, \ldots, X_{d} \uparrow_{M}$.

(ii): Noting that $L_{0}^{*}=P_{M} L^{*}=P_{M} L^{*} P_{N}$, we have

$$
\begin{aligned}
L_{0}^{*} Y_{k}-X_{k} L_{0}^{*} & =P_{M} L^{*} Y_{k}-X_{k} P_{M} L^{*} \\
& =P_{M}\left(L^{*} Y_{k}-X_{k} L^{*}\right)+\left(P_{M} X_{k}-X_{k} P_{M}\right) L^{*} .
\end{aligned}
$$

The term $L^{*} Y_{k}-X_{k} L^{*}$ belongs to $\mathcal{L}^{2 p}$ because $L$ is a $2 p$-morphism, and since $M$ is a $p$-essentially normal submodule, Proposition 4.2 implies that $P_{M} X_{k}-X_{k} P_{M} \in \mathcal{L}^{2 p}$. 
Hence $L_{0}$ is a $2 p$-morphism in $\operatorname{hom}(M, K)$. The fact that $L_{0}^{*}$ is also a $2 p$-morphism in $\operatorname{hom}(M, N)$ follows after multiplying the previous expressions on the right by $P_{N}$ and arguing the same way.

(iii): Since $M \subseteq H$ and $N=L(M) \subseteq K$ are $p$-essentially normal, Proposition 4.2 implies that their respective quotients are $p$-essentially normal as well. It remains to show that the promoted map $\dot{L}: H / M \rightarrow K / N$ is a $2 p$-morphism. For that, we identify $H / M$ with $M^{\perp} \subseteq H, K / N$ with $N^{\perp} \subseteq K$, and $\dot{L}$ with the map $\dot{L} \xi=P_{N}^{\perp} L \xi$, $\xi \in M^{\perp}, P_{N}^{\perp}$ denoting $1-P_{N}$. We have to show that $\dot{L} X_{k}^{*} P_{M}^{\perp}-Y_{k}^{*} \dot{L} P_{M}^{\perp}$ belongs to $\mathcal{L}^{2 p}$, that is,

$$
P_{N}^{\perp} L X_{k}^{*} P_{M}^{\perp}-Y_{k}^{*} P_{N}^{\perp} L P_{M}^{\perp} \in \mathcal{L}^{2 p} .
$$

The left side of (4.6) can be written

$$
P_{N}^{\perp}\left(L X_{k}^{*}-Y_{k}^{*} L\right) P_{M}^{\perp}+\left(P_{N}^{\perp} Y_{k}^{*}-Y_{k}^{*} P_{N}^{\perp}\right) L P_{M}^{\perp} .
$$

The term $L X_{k}^{*}-Y_{k}^{*} L$ belongs to $\mathcal{L}^{2 p}$ because $L$ is a $2 p$-morphism, and since $N=$ $L(M)$ has been shown to be $p$-essentially normal, Proposition 4.2 implies that $P_{N}^{\perp} Y_{k}^{*}-Y_{k}^{*} P_{N}^{\perp}=Y_{k}^{*} P_{N}-P_{N} Y_{k}^{*} \in \mathcal{L}^{2 p}$. (4.6) follows.

Remark 4.5 (Estimating the $2 p$-norm of $Y_{k} P_{N}-P_{N} Y_{k}$ ). In terms of the operator $L: H \rightarrow K$, we can estimate the $\mathcal{L}^{2 p}$-norm of $\left[Y_{k}, P_{N}\right]$ from (4.5) in the obvious way to obtain

$$
\left\|\left[Y_{k}, P_{N}\right]\right\|_{2 p} \leq(2 \pi)^{-1} \sup _{\lambda \in \Gamma}\left\|(\lambda-B)^{-1}\right\|^{2} \cdot\left\|\left[Y_{k}, B\right]\right\|_{2 p} \cdot \ell(\Gamma),
$$

$\ell(\Gamma)$ denoting the length of $\Gamma$. Noting that $B=L L^{*}$, we have $\|B\|=\|L\|^{2}$; hence the length of $\Gamma$ is $2\|L\|^{2}+2 \epsilon$, and since the minimum distance from $\Gamma$ to $\sigma(Z)$ is $\epsilon / 2$, we have $\left\|\left(\lambda-L L^{*}\right)^{-1}\right\|^{2} \leq 4 / \epsilon^{2}$. Hence

$$
\left\|\left[Y_{k}, P_{N}\right]\right\|_{2 p} \leq \frac{4\|L\|^{2}+4 \epsilon}{\pi \epsilon^{2}}\left\|\left[Z_{k}, L L^{*}\right]\right\|_{2 p} .
$$

Here, $\epsilon$ is the length of the gap between 0 and the rest of $\sigma\left(L L^{*}\right)$.

Corollary 4.6. Fix $p \in[1, \infty]$. Let $H, K$ be $p$-essentially normal Hilbert modules and let $L: H \rightarrow K$ be a $2 p$-morphism with closed range.

Let $N$ be a (closed) submodule of $L(H)$ and let $M \subseteq H$ be its pullback:

$$
M=\{\xi \in H: L \xi \in N\} .
$$

Then $L$ promotes to an isomorphism of quotients $\dot{L}: H / M \rightarrow L(H) / N$ with the property that if either $H / M$ or $L(H) / N$ is p-essentially normal, then both are $p$ essentially normal. In that event, $\dot{L}$ is a 2 -isomorphism.

Proof. In general, the promoted map $\dot{L} \in \operatorname{hom}(H / M, L(H) / N)$ is injective because $M$ is defined as the full pre-image of $N$ under $L$. It is obviously continuous and surjective; hence the closed graph theorem implies that $\dot{L}$ is an isomorphism of Hilbert modules.

Applying Theorem 4.4(i) to $M=H$, we see that $L(H)$ is a $p$-essentially normal submodule of $K$, and moreover, $L$ can be viewed as a $p$-morphism in $\operatorname{hom}(H, L(H))$. Thus by replacing $K$ with $L(H)$, we can assume that $L$ is surjective.

If $H / M$ is $p$-essentially normal, then $M$ is $p$-essentially normal by Proposition 4.2. Note that $L(M)=N$. Indeed, the inclusion $\subseteq$ is obvious and, since $L$ is surjective, every element $\eta \in N$ has the form $\eta=L(\xi)$ for some $\xi \in H$. Such a $\xi$ 
must belong to $M$ since $M$ is the pre-image of $N$. Theorem 4.4 (iii) implies that $K / N$ is $p$-essentially normal and $\dot{L}$ is a $2 p$-isomorphism.

Conversely, assume $K / N$ is $p$-essentially normal. To prove that $H / M$ is $p$ essentially normal, we identify $H / M$ with $M^{\perp}, K / N$ with $N^{\perp}$, and $\dot{L}: H / M \rightarrow$ $K / N$ with the operator

$$
\dot{L}: \xi \in M^{\perp} \mapsto P_{N}^{\perp} L \xi \in N^{\perp} .
$$

We claim that $L^{*}\left(N^{\perp}\right)=M^{\perp}$. Indeed, the preceding paragraph shows that $\dot{L}$ is an isomorphism of $M^{\perp}$ onto $N^{\perp}$; hence the adjoint $P_{M}^{\perp} L^{*} \uparrow_{N^{\perp}}$ of the operator $\dot{L}$ has range $M^{\perp}$. Noting that $L^{*}\left(N^{\perp}\right) \subseteq M^{\perp}$ simply because $L(M) \subseteq N$, it follows that $P_{M}^{\perp} L^{*} \Gamma_{N^{\perp}}=L^{*} \uparrow_{N^{\perp}}$, and therefore $L^{*}\left(N^{\perp}\right)=P_{M}^{\perp} L^{*}\left(N^{\perp}\right)=M^{\perp}$, as asserted.

The operator $d$-tuples $Y_{1}^{*}, \ldots, Y_{d}^{*}$ and $X_{1}^{*}, \ldots, X_{d}^{*}$ make $K$ and $H$ into $p$-essentially normal Hilbert modules $K^{*}$ and $H^{*}$, and $N^{\perp}, M^{\perp}$ become submodules of $K^{*}, H^{*}$, respectively. We can view $L^{*}$ as a $2 p$-morphism of $K^{*}$ to $H^{*}$ which, by the preceding paragraph, satisfies $L^{*}\left(N^{\perp}\right)=M^{\perp}$. Now Theorem 4.4 implies that $M^{\perp}$ is a $p$-essentially normal submodule of $H^{*}$. It follows that $H / M$ is $p$-essentially normal with respect to its original module structure.

Remark 4.7 (Instability of essential normality under isomorphism). Essential normality need not be preserved under isomorphism of Hilbert modules, even in dimension $d=1$. As a concrete example, let $u_{0}, u_{1}, u_{2}, \ldots$ be any sequence of real numbers with the properties

(i) $\left|u_{n+1}-u_{n}\right| \geq 1$ for infinitely many $n=0,1,2, \ldots$,

(ii) $\left|u_{0}+u_{1}+\cdots+u_{n}\right| \leq M<\infty, n=0,1,2, \ldots$,

and set

$$
\lambda_{n}=e^{u_{0}+u_{1}+\cdots+u_{n}}, \quad n=0,1,2, \ldots
$$

Let $e_{0}, e_{1}, e_{2}, \ldots$ be an orthonormal basis for a Hilbert space $H$, let $A \in \mathcal{B}(H)$ be the simple unilateral shift $A e_{n}=e_{n+1}, n \geq 0$, and let $B \in \mathcal{B}(H)$ be the unilateral weighted shift

$$
A e_{n}=e^{u_{n+1}} e_{n+1}, \quad n=0,1,2, \ldots
$$

The self-commutator $A^{*} A-A A^{*}$ is a rank-one projection, while

$$
\left(B^{*} B-B B^{*}\right) e_{n}=e^{2\left(u_{n+1}-u_{n}\right)} e_{n},
$$

so $B$ is not essentially normal by (i). On the other hand, $A$ and $B$ are similar, since one can check that the operator $L \in \mathcal{B}(H)$ defined by $L e_{n}=\lambda_{n} e_{n}$ is invertible by (ii), and satisfies $L A=B L$.

Note that the operators $A$ and $B$ make $H$ into a graded Hilbert module over $\mathbb{C}[z]$ in two ways, and in that case $L$ becomes a degree-zero isomorphism of graded Hilbert modules that does not preserve essential normality.

\section{FREDHOLM PROPERTY}

The purpose of this section is to comment on the relation between the Fredholm property, essential normality and the cohomology of the Koszul complex. We also collect an algebraic result for use in Section 6 .

Remark 5.1 (Koszul complex, Dirac operator). We briefly recall the definition and most basic properties of the Koszul complex and Dirac operator of a Hilbert module $H$ of dimension $d$ with coordinate operators $T_{1}, \ldots, T_{d}$. The reader is referred to the original sources Tay70a, Tay70b for the role of the Koszul complex in operator 
theory, and to Arv02 for more on the Dirac operator. Let $E$ be a Hilbert space of dimension $d$. For each $k=0,1, \ldots, d$ there is a Hilbert space of formal $k$-forms with coefficients in $H$,

$$
H \otimes \Lambda^{k} E,
$$

$\Lambda^{k} E$ denoting the exterior product of $k$ copies of $E$ for $k \geq 1$, and $\Lambda^{0} E$ denoting $\mathbb{C}$. Fixing an orthonormal basis $e_{1}, \ldots, e_{d}$ for $E$, one obtains creation operators $C_{1}, \ldots, C_{d}: \Lambda^{k} E \rightarrow \Lambda^{k+1} E$, defined uniquely by the requirement

$$
C_{k}: \zeta_{1} \wedge \cdots \wedge \zeta_{k} \mapsto e_{k} \wedge \zeta_{1} \wedge \cdots \wedge \zeta_{k}, \quad \zeta_{i} \in E, \quad k=1, \ldots d,
$$

and a boundary operator $B: H \otimes \Lambda^{k} E \rightarrow H \otimes \Lambda^{k+1} E$ by way of

$$
B=T_{1} \otimes C_{1}+\cdots+T_{d} \otimes C_{d} .
$$

Note that $B$ vanishes on $H \otimes \Lambda^{d} E \cong H \otimes \mathbb{C} \cong H$. This structure gives rise to a complex of Hilbert spaces

$$
0 \longrightarrow H \longrightarrow H \otimes E \longrightarrow \cdots \longrightarrow H \otimes \Lambda^{d-1} E \longrightarrow H \otimes \Lambda^{d} E \longrightarrow 0 .
$$

One can assemble the various $H \otimes \Lambda^{k} E$ into a single graded Hilbert space $H \otimes \Lambda E$ by forming the direct sum of spaces of $k$-forms

$$
H \otimes \Lambda E=\left(H \otimes \Lambda^{0} E\right) \oplus \cdots \oplus\left(H \otimes \Lambda^{d} E\right),
$$

thereby making $B$ into a graded operator of degree one in $\mathcal{B}(H \otimes \Lambda E)$ that satisfies $B^{2}=0$. This structure is called the Koszul complex of $H$.

The Dirac operator of $H$ is the operator $D=B+B^{*}$. We are suppressing the Clifford structure attached to $D$, which is incidental to our needs here. The cohomology of the Koszul complex is related to the Dirac operator as follows: $B\left(H \otimes \Lambda^{k} E\right)$ has finite codimension in $\operatorname{ker}\left(B \uparrow_{H \otimes \Lambda^{k+1} E}\right)$, for every $k=0,1, \ldots, d$ iff $D$ is a self-adjoint Fredholm operator [Arv02.

We say that $H$ has the Fredholm property when these equivalent conditions are satisfied. The following general result gives a sufficient condition for Fredholmness that is relatively easy to check in specific examples. While it is a small variation of a result of Curto Cur81, we include a proof for completeness.

Proposition 5.2. Let $H$ be an essentially normal Hilbert module such that $T_{1} H+$ $\cdots+T_{d} H$ is a closed subspace of finite codimension in $H$. Then $H$ has the Fredholm property.

Proof. The hypothesis on the space $T_{1} H+\cdots+T_{d} H$ is equivalent to the assertion that $T_{1} T_{1}^{*}+\cdots+T_{d} T_{d}^{*}$ is a self-adjoint Fredholm operator. We show that the latter property, taken together with essential normality, implies that the Dirac operator $D$ is Fredholm.

Let $B=T_{1} \otimes C_{1}+\cdots+T_{d} \otimes C_{d}$ be the boundary operator. Then $D=B+B^{*}$ and, since $T_{j}$ commutes with $T_{k}$ and $C_{j}$ anticommutes with $C_{k}$, one has $B^{2}=0$. Therefore

$$
D^{2}=\left(B+B^{*}\right)^{2}=B^{*} B+B B^{*}=\sum_{k, j=1}^{d} T_{k}^{*} T_{j} \otimes C_{k}^{*} C_{j}+\sum_{k, j=1}^{d} T_{j} T_{k}^{*} \otimes C_{j} C_{k}^{*} .
$$

Using $C_{j} C_{k}^{*}=\delta_{j k} \mathbf{1}-C_{k}^{*} C_{j}$, we can write the second term on the right as

$$
F \otimes \mathbf{1}-\sum_{k, j=1}^{d} T_{j} T_{k}^{*} \otimes C_{k}^{*} C_{j}
$$


where $F=T_{1} T_{1}^{*}+\cdots+T_{d} T_{d}^{*}$, so that

$$
D^{2}=F \otimes \mathbf{1}+\sum_{k, j=1}^{d}\left[T_{k}^{*}, T_{j}\right] \otimes C_{k}^{*} C_{j} .
$$

Since $F \otimes \mathbf{1}$ is a Fredholm operator and each summand in the second term is compact by hypothesis, it follows that $D^{2}$ is a Fredholm operator. Since $D$ is self-adjoint, $D$ itself must be a Fredholm operator.

Remark 5.3 (Cohomology type, the final three terms). Let $S=G \otimes \mathbb{C}^{r}$ be a standard Hilbert module of rank $r$. If $G$ is essentially normal, then so is $S$, and therefore Proposition 5.2 implies that $S$ has the Fredholm property. Indeed, it is quite easy to show in that case that the cohomology type of $S$ (by which we mean the sequence of Betti numbers $\left(\beta_{0}, \beta_{1}, \ldots, \beta_{d}\right)$ of the Koszul complex of $\left.S\right)$ is $(0,0, \ldots, 0, r)$, the cohomology type of the free algebraic module $\mathcal{A} \otimes \mathbb{C}^{r}$ of rank $r$. Thus, essentially normal standard Hilbert modules have the same cohomology type as free modules.

In particular, the behavior of the boundary operator of the Koszul complex of a standard Hilbert module is specified at every stage $S \otimes \Lambda^{k} E, 0 \leq k \leq d$. In this paper we shall only have to refer to the last three terms of the Koszul complex

$$
\begin{aligned}
& B_{d-2}: S \otimes \Lambda^{d-2} E \rightarrow S \otimes \Lambda^{d-1} E, \\
& B_{d-1}: S \otimes \Lambda^{d-1} E \rightarrow S \otimes \Lambda^{d} E=S,
\end{aligned}
$$

for graded Hilbert modules whose last two Betti numbers are $0, r$. In such cases, the two boundary operators satisfy a) $\operatorname{ran} B_{d-2}=\operatorname{ker} B_{d-1}$ and b) $\operatorname{ran} B_{d-1}$ is a closed subspace of $S$ of finite codimension $r$.

We now point out how the assertions a) and b) can be cast into a more concrete form for graded modules $H=H_{0} \oplus H_{1} \oplus \cdots$ with coordinate operators $T_{1}, \ldots, T_{d}$ that satisfy $0<\operatorname{dim} H_{0}<\infty$ and

$$
H_{n+1}=T_{1} H_{n}+\cdots+T_{d} H_{n}, \quad n=0,1,2, \ldots
$$

Let us first consider b). Note that $H \otimes \Lambda^{d-1} E$ can be identified with the direct sum $d \cdot H$ of $d$ copies of $H$ in such a way that, up to sign, the boundary map $B_{d-1}: H \otimes \Lambda^{d-1} E \rightarrow H$ becomes identified with the row operator

$$
\bar{T}:\left(\xi_{1}, \ldots, \xi_{d}\right) \in d \cdot H \mapsto T_{1} \xi_{1}+\cdots+T_{d} \xi_{d} \in H .
$$

The range of this map is dense in $H_{1} \oplus H_{2} \oplus \cdots=H_{0}^{\perp}$ and will be of finite (algebraic) codimension in $H \Longleftrightarrow T_{1} H+\cdots+T_{d} H$ is closed $\Longleftrightarrow T_{1} T_{1}^{*}+\cdots+T_{d} T_{d}^{*}$ is a selfadjoint Fredholm operator. Note too that since the orthocomplement of $\bar{T}(d \cdot H)$ is the space $H_{0} \neq\{0\}$, it follows that the complex (5.1) cannot be exact at the final term $H \otimes \Lambda^{d} E$.

In more explicit terms, a) makes the assertion that for every $d$-tuple of vectors $\xi_{1}, \ldots, \xi_{d}$ in $H$ with the property $T_{1} \xi_{1}+\cdots+T_{d} \xi_{d}=0$, there is a skew-symmetric array $\eta_{i j}=-\eta_{j i} \in H, 1 \leq i, j \leq d$, such that

$$
\xi_{k}=\sum_{j=1}^{d} T_{j} \eta_{j k}, \quad k=1,2, \ldots, d .
$$

We require the representation (5.2) in Proposition 6.1 below. For now, we simply note that if $\xi_{1}, \ldots, \xi_{d} \in H_{n}$ are homogeneous vectors of degree $n=0,1,2, \ldots$ satisfying $T_{1} \xi_{1}+\cdots+T_{d} \xi_{d}=0$, then since each operator $T_{j}$ is of degree one, the 
vectors $\eta_{i j}$ of (5.2) must belong to $H_{n-1}$. In particular, for the case $n=0$ we have $H_{-1}=\{0\}$, and therefore

$$
\xi_{1}, \ldots, \xi_{d} \in H_{0}, \quad T_{1} \xi_{1}+\cdots+T_{d} \xi_{d}=0 \Longrightarrow \xi_{1}=\cdots=\xi_{d}=0 .
$$

\section{KERNELS OF DEGREE 1}

Let $S=G \otimes E$ be a standard Hilbert module and let $L: d \cdot S \rightarrow S$ be the row operator

$$
L\left(\xi_{1}, \ldots, \xi_{d}\right)=Z_{1} \xi_{1}+\cdots+Z_{d} \xi_{d}, \quad \xi_{1}, \ldots, \xi_{d} \in S .
$$

We have seen that the range of $L$ is a closed submodule of $S, \operatorname{ran} L=S_{1} \oplus S_{2} \oplus \cdots$, and in particular ran $L$ is of degree 1 . The kernel of $L$ is a graded submodule of $d \cdot S$ that will occupy a central position in the following section. In this section we calculate its degree.

Proposition 6.1. The kernel $K=\operatorname{ker} L$ of the row operator of (6.1) is a graded submodule of $d \cdot S$ of degree 1 .

Proof. That $K=K_{0} \oplus K_{1} \oplus \cdots$ is graded is clear from the fact that $L$ maps $(d \cdot S)_{n}$ into $S_{n+1}$ for every $n=0,1,2, \ldots$, and (5.3) asserts that $K_{0}=\{0\}$. Hence $K=K_{1} \oplus K_{2} \oplus \cdots$.

Since the inclusion $Z_{j} K_{n} \subseteq K_{n+1}, 1 \leq j \leq d$, is obvious, we have to prove

$$
K_{n+1} \subseteq Z_{1} K_{n}+\cdots+Z_{d} K_{n}, \quad n \geq 1 .
$$

To that end, choose $\xi \in K_{n+1}$, say $\xi=\left(\xi_{1}, \ldots, \xi_{d}\right)$ with $\xi_{j} \in S_{n+1}$ satisfying $Z_{1} \xi_{1}+\cdots+Z_{d} \xi_{d}=0$. Formula (5.2) implies that there is an antisymmetric array $\eta_{i j}=-\eta_{j i} \in S_{n}, 1 \leq i, j \leq d$, such that $\xi_{j}=\sum_{i} Z_{i} \eta_{i j}$ for $j=1, \ldots, d$. We consider the array $\left(\eta_{i j}\right)_{1 \leq i<j \leq d}$ as an element of $q \cdot S$, where $q=d(d-1) / 2$. This $q$-tuple of vector polynomials is homogeneous of degree $n$, and therefore it belongs to $Z_{1}(q \cdot S)_{n-1}+\cdots+Z_{d}(q \cdot S)_{n-1}$. Thus we can find a set of $d$ such arrays $\left(\zeta_{i j}^{1}\right)_{1 \leq i<j \leq d}, \ldots,\left(\zeta_{i j}^{d}\right)_{1 \leq i<j \leq d} \in q \cdot S$, each component of which is homogeneous of degree $n-1$, such that

$$
\eta_{i j}=\sum_{p=1}^{d} Z_{p} \zeta_{i j}^{p}, \quad 1 \leq i<j \leq d .
$$

Setting $\zeta_{i j}^{p}=-\zeta_{j i}^{p}$ for $i>j$ and $\zeta_{i i}^{p}=0$ for $1 \leq i \leq d$, we obtain antisymmetric arrays $\left(\zeta_{i j}^{1}\right), \ldots,\left(\zeta_{i j}^{d}\right)$ which, since $\eta_{i j}$ is also antisymmetric, satisfy

$$
\eta_{i j}=\sum_{p=1}^{d} Z_{p} \zeta_{i j}^{p}, \quad 1 \leq i, j \leq d .
$$

It follows that

$$
\xi_{i}=\sum_{j, p=1}^{d} Z_{j} Z_{p} \zeta_{i j}^{p},
$$

and therefore $\xi$ can be written as a linear combination

$$
\xi=\left(\xi_{1}, \ldots, \xi_{d}\right)=Z_{1} \omega_{1}+\cdots+Z_{d} \omega_{d}
$$

of elements

$$
\omega_{p}=\left(\sum_{j} Z_{j} \zeta_{1 j}^{p}, \cdots, \sum_{j} Z_{j} \zeta_{d j}^{p}\right), \quad p=1, \ldots, d
$$


Each $\omega_{p}$ belongs to $d \cdot S_{n}$, and we have

$$
L \omega_{p}=\sum_{i, j=1}^{d} Z_{i} Z_{j} \zeta_{i j}^{p}=0,
$$

because for $p$ fixed, $Z_{i} Z_{j} \zeta_{i j}^{p}$ is antisymmetric in $i$ and $j$. Hence $\omega_{p} \in K_{n}$, so that (6.2) exhibits $\xi$ as an element of $Z_{1} K_{n}+\cdots+Z_{d} K_{n}$.

\section{Stability of QUOTIENTS UNDER ShIFTING}

One can shift a graded Hilbert module $H=H_{0} \oplus H_{1} \oplus \cdots$ to the left to obtain another graded Hilbert module $H^{\prime}=H_{1} \oplus H_{2} \oplus \cdots$, in which the grading on $H^{\prime}$ is given by $H_{n}^{\prime}=H_{n+1}, n \geq 0$. One can also view $H^{\prime}$ as a submodule of $H$ of codimension $\operatorname{dim} H_{0}$, with a grading different from the inherited grading. In this section we show that in an appropriate sense, the class of graded Hilbert modules that are isomorphic to quotients $(G \otimes E) / M$ of standard Hilbert modules based on a fixed graded completion $G$ is stable under this operation of left-shifting.

The examples of Remark 4.7 show that, in general, isomorphisms of Hilbert modules may not preserve essential normality. We first strengthen the notion of isomorphism so as to get rid of this anomaly.

Definition 7.1 (Strong equivalence). Let $H$ and $K$ be Hilbert modules with respective operator $d$-tuples $X_{1}, \ldots, X_{d}$ and $Y_{1}, \ldots, Y_{d} . H$ and $K$ are said to be strongly equivalent if there is an isomorphism of Hilbert modules $L: H \rightarrow K$ such that $L X_{k}^{*}-Y_{k}^{*} L$ is compact for every $k=1, \ldots, d$.

In the category of graded Hilbert modules, strong isomorphisms are required to preserve degree: $L\left(H_{n}\right)=K_{n}, n=0,1, \ldots$.

Remark 7.2 (Strong equivalence and essential normality). Strong equivalence is obviously an equivalence relation. More significantly, if an essentially normal Hilbert module $H$ is strongly equivalent to a Hilbert module $K$, then $K$ must be essentially normal as well. Indeed, choosing $L: H \rightarrow K$ as in Definition 7.1, it follows that $\left[X_{k}, L^{*} L\right]=X_{k} L^{*} L-L^{*} L X_{k}$ is compact, hence $\left[X_{k},\left(L^{*} L\right)^{-1}\right]$ is compact, and writing $Y_{k}=L X_{k} L^{-1}$, we find that for $1 \leq j, k \leq d$,

$$
\begin{aligned}
Y_{k} Y_{j}^{*}-Y_{j}^{*} Y_{k} & =L X_{k}\left(L^{*} L\right)^{-1} X_{j}^{*} L^{*}-L^{*-1} X_{j}^{*} L^{*} L X_{k} L^{-1} \\
& =L\left[X_{k},\left(L^{*} L\right)^{-1}\right]+L^{*-1}\left(X_{k} X_{j}^{*}-X_{j}^{*} X_{k}\right) L^{-1} \in \mathcal{K} .
\end{aligned}
$$

Theorem 7.3 (Stability of $G$-quotients). Let $G$ be an essentially normal graded completion and let $H=H_{0} \oplus H_{1} \oplus \cdots$ be a graded Hilbert module that is strongly equivalent to a $G(n)$-quotient for some $n \geq 2$. Then the shifted module $H^{\prime}=$ $H_{1} \oplus H_{2} \oplus \cdots$ is strongly equivalent to a $G(n-1)$-quotient.

Proof. By hypothesis, there is a standard Hilbert module $S=G \otimes E$ based on $G$ and a graded submodule $M \subseteq S$ of degree $n$ such that $H$ is strongly equivalent to $S / M$. By Remark 7.2 we may, without loss of generality, take $H=S / M$ and $H_{k}=S_{k} / M_{k}, k=0,1,2, \ldots$ Letting $Z_{1}, \ldots, Z_{d}$ be the coordinate operators of $S$, one sees that the shifted module $H^{\prime}$ is given by

$$
H^{\prime}=S_{1} / M_{1} \oplus S_{2} / M_{2} \oplus \cdots=\left(Z_{1} S+\cdots+Z_{d} S\right) /\left(M_{1} \oplus M_{2} \oplus \cdots\right) .
$$


We have to show that there is another standard Hilbert module $S^{\prime}=G \otimes E^{\prime}$ based on $G$, a graded submodule $M^{\prime} \subseteq S^{\prime}$ of degree $n-1$, and a strong isomorphism $L$ that maps $S^{\prime} / M^{\prime}$ to $H^{\prime}$.

Let $S^{\prime}=d \cdot S=G \otimes d \cdot E$ be the direct sum of $d$ copies of $S$, and let $L: d \cdot S \rightarrow S$ be the row operator $\left(Z_{1}, \ldots, Z_{d}\right)$ :

$$
L\left(\xi_{1}, \ldots, \xi_{d}\right)=Z_{1} \xi_{1}+\cdots+Z_{d} \xi_{d}, \quad \xi_{1}, \ldots, \xi_{d} \in S
$$

Since $G$ is a graded completion, the range of $L$ is the closed submodule of $S$ given by $Z_{1} S+\cdots+Z_{d} S=S_{1} \oplus S_{2} \oplus \cdots$. Let $M^{\prime}$ be the pullback of $M$,

$$
M^{\prime}=\{\zeta \in d \cdot S: L \zeta \in M\} .
$$

We have $L\left(S_{k}^{\prime}\right)=S_{k+1}$ for $k=0,1,2, \ldots$; hence $M^{\prime}=M_{0}^{\prime} \oplus M_{1}^{\prime} \oplus \cdots$ is a graded submodule of $S^{\prime}=d \cdot S$ that contains the kernel of $L$ and satisfies

$$
L\left(M_{k}^{\prime}\right)=M_{k+1}, \quad k=0,1,2, \ldots
$$

Therefore $L\left(M^{\prime}\right)=M \cap L\left(S^{\prime}\right)=M_{1} \oplus M_{2} \oplus \cdots$. It follows that $L$ promotes to an isomorphism of Hilbert modules $\dot{L}: S^{\prime} / M^{\prime} \rightarrow H^{\prime}$.

Since $G$ is essentially normal the commutators $Z_{j}^{*} Z_{k}-Z_{k} Z_{j}^{*}$ are compact; therefore since $L=\left(Z_{1}, \ldots, Z_{d}\right)$ is a row operator whose components $Z_{k}$ commute modulo $\mathcal{K}$ with $Z_{1}^{*}, \ldots, Z_{d}^{*}, L$ must be an $\infty$-morphism. At this point we can apply the last sentence of Corollary 4.6 to conclude that $\dot{L}$ implements a strong isomorphism of graded Hilbert modules $S^{\prime} / M^{\prime} \cong H^{\prime}$.

It remains to show that the pullback $M^{\prime}$ is of degree $n-1$, i.e.,

$$
Z_{1} M_{k}^{\prime}+\cdots+Z_{d} M_{k}^{\prime}=M_{k+1}^{\prime}, \quad k \geq n-1,
$$

and

$$
Z_{1} M_{n-2}^{\prime}+\cdots+Z_{d} M_{n-2}^{\prime} \neq M_{n-1}^{\prime} .
$$

The inclusion $\subseteq$ of (7.2) follows from the fact that $M^{\prime}$ is a graded submodule of $S^{\prime}$. To prove the opposite inclusion, let $k \geq n-1$ and choose $\xi \in M_{k+1}^{\prime}$. Using (7.1) and the fact that $M$ is of degree $n$, we have

$$
\begin{aligned}
L \xi \in M_{k+2} & =Z_{1} M_{k+1}+\cdots+Z_{d} M_{k+1}=Z_{1} L\left(M_{k}^{\prime}\right)+\cdots+Z_{d} L\left(M_{k}^{\prime}\right) \\
& =L\left(Z_{1} M_{k}^{\prime}+\cdots+Z_{d} M_{k}^{\prime}\right),
\end{aligned}
$$

and therefore $\xi \in Z_{1} M_{k}^{\prime}+\cdots+Z_{d} M_{k}^{\prime}+\operatorname{ker} L$. Noting that $\operatorname{ker} L$ is a graded submodule,

$$
\operatorname{ker} L=K_{1} \oplus K_{2} \oplus \cdots,
$$

and that both $\xi$ and the space $Z_{1} M_{k}^{\prime}+\cdots+Z_{d} M_{k}^{\prime}$ are homogeneous of degree $k+1$, we must have $\xi \in\left(Z_{1} M_{k}^{\prime}+\cdots+Z_{d} M_{k}^{\prime}\right)+K_{k+1}$. Since $k+1 \geq n \geq 2$, Proposition 6.1 implies that $K_{k+1}=Z_{1} K_{k}+\cdots+Z_{d} K_{k}$, so that

$$
\xi \in Z_{1}\left(M_{k}^{\prime}+K_{k}\right)+\cdots+Z_{d}\left(M_{k}^{\prime}+K_{k}\right) .
$$

Finally, since $M^{\prime}$ contains $\operatorname{ker} L$ we have $K_{k} \subseteq M_{k}^{\prime}$, so that

$$
\xi \in Z_{1}\left(M_{k}^{\prime}+K_{k}\right)+\cdots+Z_{d}\left(M_{k}^{\prime}+K_{k}\right)=Z_{1} M_{k}^{\prime}+\cdots+Z_{d} M_{k}^{\prime},
$$

and (7.2) is established.

To prove (7.3) we proceed contrapositively. If (7.3) fails, then

$$
M_{n-1}^{\prime}=Z_{1} M_{n-2}^{\prime}+\cdots+Z_{d} M_{n-2}^{\prime}
$$


and, after applying $L$ and using $L Z_{j}=Z_{j} L$ for $j=1, \ldots, d$, we obtain

$$
\begin{aligned}
M_{n-1} & =L\left(M_{n-1}^{\prime}\right)=Z_{1} L\left(M_{n-2}^{\prime}\right)+\cdots+Z_{d} L\left(M_{n-2}^{\prime}\right) \\
& =Z_{1} M_{n-2}+\cdots+Z_{d} M_{n-2} .
\end{aligned}
$$

This implies that $\operatorname{deg} M \leq n-1$, contradicting $\operatorname{deg} M=n$.

\section{LiNEARIZATIONS}

We now assemble the preceding results to deduce the linearization result alluded to in the introduction and discuss its implications for the issue of essential normality. Throughout the section, $G$ will denote an essentially normal graded completion of $\mathcal{A}$.

Theorem 8.1 (Linearization of $G$-quotients). Let $M \subseteq G \otimes E$ be a graded submodule of a standard Hilbert module based on $G$, let $H=(G \otimes E) / M$ be its quotient, and consider the natural grading of $H=H_{0} \oplus H_{1} \oplus \cdots$. If the degree $n=\operatorname{deg} M$ of $M$ is larger than 1, then the shifted module

$$
H_{n-1} \oplus H_{n} \oplus H_{n+1} \oplus \cdots
$$

is strongly equivalent to the quotient $S^{\prime} / M^{\prime}$ of another standard Hilbert module $S^{\prime}=G \otimes E^{\prime}$ based on $G$ by a graded submodule $M^{\prime}$ of degree 1 .

Proof. The Hilbert module (8.1) is seen to be strongly equivalent to a $G(1)$-quotient after a straightforward iteration of Theorem 7.3 through $n-1$ steps. We omit the details.

It is convenient to introduce the following terminology.

Definition 8.2. Let $H=(G \otimes E) / M$ be a $G$-quotient. By a linearization of $H$ we mean a $G$-quotient $H^{\prime}=\left(G \otimes E^{\prime}\right) / M^{\prime}$ with the following properties:

(i) $M^{\prime}$ is a degree 1 graded submodule of $G \otimes E^{\prime}$.

(ii) $H^{\prime}$ is strongly equivalent to a closed submodule of finite codimension in $H$.

Remark 8.3 (Nonuniqueness of linearizations). Let $H=H_{0} \oplus H_{1} \oplus \cdots$ be a $G(n)$ quotient for some $n \geq 2$. Theorem 8.1 asserts that the $(n-1)$-shifted submodule (8.1) is strongly equivalent to a $G(1)$-quotient $\left(G \otimes E^{\prime}\right) / M^{\prime}$. If some other $G(1)$ quotient $\left(G \otimes E^{\prime \prime}\right) / M^{\prime \prime}$ is strongly equivalent to the module of (8.1), then of course the two $G(1)$-quotients $\left(G \otimes E^{\prime}\right) / M^{\prime}$ and $\left(G \otimes E^{\prime \prime}\right) / M^{\prime \prime}$ must be strongly equivalent. Thus one would like to know the extent to which a $G(1)$-quotient is uniquely determined by its strong equivalence class. In the case where $G$ is the space $H^{2}$ of the $d$-shift, one knows that the unitary equivalence class of quotient modules such as $\left(H^{2} \otimes E\right) / M$ uniquely determines the pair $M \subseteq H^{2} \otimes E$ up to unitary equivalence once one imposes a natural minimality requirement Arv98. On the other hand, even for this case $G=H^{2}$, we know of no classification of $G$-quotients (even $G(1)$-quotients) up to strong equivalence.

In a sense, Theorem 8.1 provides the simplest linearization of a $G(n)$-quotient for $n \geq 2$. Indeed, one can ask what happens when one shifts the module of (8.1) further to the left. It is not hard to show that further shifting does not reduce $G(1)$-quotients to $G(0)$-quotients. Rather, it simply leads to an infinite sequence of new Hilbert modules

$$
H_{k} \oplus H_{k+1} \oplus H_{k+2} \oplus \cdots, \quad k=n-1, n, n+1, \ldots,
$$


each of which is strongly equivalent to a $G(1)$-quotient. We omit the proof since we do not require that result. However, from these remarks it is apparent that every $G$-quotient has infinitely many linearizations.

Theorem 8.1 has the following consequence, which reduces the problem of establishing essential normality of quotients to that of establishing essential normality of linearized quotients:

Corollary 8.4. Let $G$ be an essentially normal graded completion, and let $H=$ $(G \otimes E) / M$ be a G-quotient. Then $H$ has linearizations, and the following are equivalent:

(i) $H$ is essentially normal.

(ii) Some linearization of $H$ is essentially normal.

(iii) Every linearization of $H$ is essentially normal.

Proof. Let $H_{0} \subseteq H$ be a closed submodule of a Hilbert module such that $H / H_{0}$ is finite-dimensional. Since the projection on $H_{0}$ is a finite-rank perturbation of the identity, Proposition 4.2 implies that $H$ is essentially normal iff $H_{0}$ is essentially normal. Thus, the existence of linearizations follows from the statement of Theorem 8.1. Since strong equivalence preserves essential normality of Hilbert modules, the equivalence of (i) - (iii) follows.

\section{Structure of Linearized QUOTIEnts}

Linearized quotients admit a particularly concrete description rooted in basic algebra, especially in the case where $G$ has maximum symmetry in the sense of Appendix A. In particular, the problem of establishing essential normality for graded quotients $\left(H^{2} \otimes E\right) / M$ is reduced to the problem of establishing essential normality for these concrete examples. We spell that out in this section, concentrating on the maximally symmetric cases. It is exasperating that the problem of establishing essential normality for these examples remains open.

Throughout the section, $S=G \otimes E$ denotes a standard Hilbert module based on a graded completion $G$ of the polynomial algebra $\mathcal{A}$. Note first that $\mathcal{A} \otimes E$ is a graded module over the polynomial algebra $\mathcal{A}$ that has $S$ as it closure. More explicitly, the tensor product of the $G$-inner product on $\mathcal{A}$ and the given inner product on $E$ gives rise to an inner product on $\mathcal{A} \otimes E$, and the completion of $\mathcal{A} \otimes E$ in that norm is the Hilbert module $S=G \otimes E$. Throughout the section, it will be convenient to view elements $f$ of $\mathcal{A} \otimes E$ as $E$-valued vector polynomials

$$
z \in \mathbb{C}^{d} \mapsto f(z) \in E .
$$

The homogeneous summand $S_{n}$ is the space $\mathcal{A}_{n} \otimes E$ of homogeneous polynomials of degree $n$ that take values in $E, n=0,1,2, \ldots$.

For every linear subspace $V \subseteq E$, the closure of $\mathcal{A} \otimes V$ in $S$ is a (necessarily graded) reducing submodule of $S$; and by Remark 3.4, every reducing submodule of $S$ is obtained in this way from a uniquely determined subspace $V \subseteq E$. We begin by summarizing the elementary features of the "pointwise" description of these reducing submodules.

Proposition 9.1. Let $V$ be a linear subspace of $E$. The space $\mathcal{V}$ of all vector polynomials $f \in \mathcal{A} \otimes E$ that satisfy

$$
f(z) \in V, \text { for all } z=\left(z_{1}, \ldots, z_{d}\right) \in \mathbb{C}^{d}
$$


is graded in that $\mathcal{V}=\mathcal{V}_{0} \dot{+} \mathcal{V}_{1} \dot{+} \mathcal{V}_{2} \dot{+} \cdots$, where $\mathcal{V}_{n}=\mathcal{A}_{n} \otimes V$ consists of all homogeneous polynomials of degree $n$ that satisfy (9.1). The closure of $\mathcal{V}$ in $S=$ $G \otimes E$ is the reducing submodule $G \otimes V$.

Proof. Let $f \in \mathcal{A} \otimes E$ be a polynomial satisfying (9.1). Then for each $r \geq 0$, the scaled polynomial $f_{r}(z)=f(r z)$ also satisfies (9.1). After noting the Taylor expansion of $f$,

$$
f(r z)=f_{0}(z)+r f_{1}(z)+r^{2} f_{2}(z)+\cdots, \quad z \in \mathbb{C}^{d},
$$

and carrying out obvious differentiations with respect to $r$, one finds that $f_{n}(z) \in V$, $z \in \mathbb{C}^{d}, n \geq 0$; hence each homogeneous polynomial $f_{n}$ satisfies (9.1). By the preceding remarks, homogeneous polynomials of degree $n$ that satisfy (9.1) are the elements of $\mathcal{A}_{n} \otimes V$. Since $f_{0}+f_{1}+\cdots=f$, it follows that $f$ belongs to $\mathcal{V}_{0}+\mathcal{V}_{1}+\cdots$.

It follows that the closure of $\mathcal{V}$ in the norm of $S=G \otimes E$ is a graded submodule with homogeneous summands $\mathcal{V}_{n}=\mathcal{A}_{n} \otimes V, n=0,1,2, \ldots$. Hence it is $G \otimes V$.

Remark 9.2 (The differential operator $D_{G}: \mathcal{A} \otimes E \rightarrow \mathcal{A} \otimes d \cdot E$ ). Fix a graded completion $G$ and consider the adjoints $Z_{k}^{*}, 1 \leq k \leq d$, of the coordinate operators of $G$. Recalling that $G_{n}=\mathcal{A}_{n}$ and that $Z_{k}^{*} G_{n} \subseteq G_{n-1}$ for $n \geq 1$ and $Z_{k}^{*} G_{0}=\{0\}$, it follows that each $Z_{k}^{*}$ leaves $\mathcal{A}$ invariant and carries $\mathcal{A}_{n}$ into $\mathcal{A}_{n-1}$. It goes without saying that, while the action of $Z_{k}$ on $\mathcal{A}$ depends only on the algebraic structure of $\mathcal{A}$ (and the specified basis for $\mathcal{A}_{1}$ ), the operators $Z_{k}^{*}$ depend strongly on the inner product associated with $G$.

For higher multiplicity standard Hilbert modules $S=G \otimes E$ based on $G$, the adjoints of the coordinate operators of $S$ are the higher multiplicity versions $Z_{k}^{*} \otimes \mathbf{1}_{E}$ of the corresponding operators on $G$. We will continue to abuse notation by writing $Z_{k}$ and $Z_{k}^{*}$ for the coordinate operators of $S$ and their adjoints, whenever it does not lead to confusion. Thus, each $Z_{k}^{*}$ acts as a linear operator of degree -1 on the graded algebraic module $\mathcal{A} \otimes E$. We find it convenient to think of $Z_{k}^{*}$ as a generalized first order differential operator (for example, see (9.3) below).

If we realize $d \cdot(\mathcal{A} \otimes E)=\mathcal{A} \otimes d \cdot E$ as the space of $d$-tuples $\left(f, \ldots, f_{d}\right)$ of vector polynomials $f_{k} \in \mathcal{A} \otimes E$, then we can define a linear operator $D_{G}: \mathcal{A} \otimes E \rightarrow \mathcal{A} \otimes d \cdot E$ as follows:

$$
D_{G} f=\left(Z_{1}^{*} f, \ldots, Z_{d}^{*} f\right), \quad f \in \mathcal{A} \otimes E .
$$

The case where $G=H^{2}$ is the space of the $d$-shift is noteworthy, since in that case the adjoints of the coordinate operators of $H^{2}$ act on polynomials by way of

$$
Z_{k}^{*} f(z)=(N+\mathbf{1})^{-1} \frac{\partial f}{\partial z_{k}}(z), \quad z \in \mathbb{C}^{d},
$$

where $N$ is the number operator of $H^{2}$. It follows that the action of $D_{H^{2}}$ on vector polynomials in $\mathcal{A} \otimes E$ is given by

$$
D_{H^{2}} f(z)=(N+\mathbf{1})^{-1}\left(\frac{\partial f}{\partial z_{1}}(z) \ldots, \frac{\partial f}{\partial z_{d}}(z)\right)=(N+\mathbf{1})^{-1} \nabla f(z),
$$

$\nabla$ denoting the classical gradient operator. More generally, if $G$ is a graded completion associated with a maximally symmetric inner product as in Appendix A, then $D_{G}$ takes the form

$$
D_{G} f(z)=u(N) \nabla f(z), \quad z \in \mathbb{C}^{d},
$$

where $u$ is an appropriate bounded function in $C[0, \infty)$. 
In order to keep the statement of results as simple as possible, we confine attention to maximally symmetric graded completions $G$, so that the operators $D_{G}: \mathcal{A} \otimes E \rightarrow \mathcal{A} \otimes d \cdot E$ have the form (9.5). We seek a concrete description of all $G(1)$-quotients in elementary terms (Theorem 9.3). At the end of the section we indicate how that description should be modified for more general graded completions.

We begin by giving a purely algebraic description of a family of subspaces of $\mathcal{A} \otimes E$ that are invariant under the action of the differentiation operators $\frac{\partial}{\partial z_{1}}, \ldots, \frac{\partial}{\partial z_{d}}$. Let $E$ be a finite-dimensional vector space, let $V$ be a linear subspace of the direct sum $d \cdot E$ of $d$ copies of $E$, and consider the space $\mathcal{E}_{V}$ of all polynomials $f \in \mathcal{A} \otimes E$ with the property:

$$
\nabla f(z) \in V, \quad z=\left(z_{1}, \ldots, z_{d}\right) \in \mathbb{C}^{d} .
$$

One finds that $\mathcal{E}_{V}$ is a graded vector space,

$$
\mathcal{E}_{V}=\mathcal{E}_{V}(0) \dot{+} \mathcal{E}_{V}(1) \dot{+} \mathcal{E}_{V}(2) \dot{+} \cdots,
$$

in which $\mathcal{E}_{V}(n)$ denotes the space of all homogeneous polynomials of degree $n$ that satisfy (9.6) (the argument being a minor variation on the proof of Proposition 9.1). One also has

$$
\frac{\partial}{\partial z_{k}} \mathcal{E}_{V}(n) \subseteq \mathcal{E}_{V}(n-1), \quad n=1,2, \ldots, \quad k=1, \ldots, d
$$

and $\mathcal{E}_{0}$, the space of constant polynomials, is annihilated by $\frac{\partial}{\partial z_{1}}, \ldots, \frac{\partial}{\partial z_{d}}$.

Theorem 9.3. Let $S=G \otimes E$ be a standard Hilbert module based on a maximally symmetric graded completion of $\mathcal{A}$ and let $Z_{1}^{*}, \ldots, Z_{d}^{*}$ be the adjoints of the coordinate multiplications of $S$. Then $Z_{k}^{*} \mathcal{E}_{V} \subseteq \mathcal{E}_{V}$; hence the orthocomplement of $\mathcal{E}_{V}$ in $S$ is a graded submodule of $S$, and the closure of $\mathcal{E}_{V}$ in $S$ is identified with the quotient module

$$
H_{V}=S / \mathcal{E}_{V}^{\perp}
$$

This quotient has the property that its submodule $M=\mathcal{E}_{V}^{\perp}$ is a degree-one graded submodule of $Z_{1} S+\cdots+Z_{d} S$.

Conversely, every degree-one graded submodule $M \subseteq Z_{1} S+\cdots+Z_{d} S$ has the above form $M=\mathcal{E}_{V}^{\perp}$ and its quotient $S / M$ has the form $H_{V}$, for a uniquely determined linear subspace $V \subseteq d \cdot E$.

Proof. We begin with the observation that for any standard Hilbert module $S=$ $G \otimes E$ based on a maximally symmetric graded completion $G$, the restriction of $Z_{k}^{*}$ to $\mathcal{A} \otimes E$ has the form

$$
Z_{k}^{*}=u(N) \frac{\partial}{\partial z_{k}},
$$

where $u(N)$ denotes a bounded function of the number operator $N$ that depends on the inner product of $G$ (see formula (9.5)). It follows that for each $n \geq 0$, the restriction of $Z_{k}^{*}$ to $\mathcal{A}_{n} \otimes E$ is a scalar multiple of $\frac{\partial}{\partial z_{k}}$.

In view of (9.7), the closure of $\mathcal{E}_{V}$ becomes a graded subspace of $S$ that is invariant under $Z_{1}^{*}, \ldots, Z_{d}^{*}$. Thus we may conclude that a) $M=\mathcal{E}_{V}^{\perp}$ is a graded submodule of $S$, and b) the quotient $S / M$ is identified with the closure of $\mathcal{E}_{V}$, so that the coordinate operators of $S / M$ are identified with the compressions of $Z_{1}, \ldots, Z_{d}$ to $\overline{\mathcal{E}_{V}}$. 
To see that the graded submodule $M=\mathcal{E}_{V}^{\perp}$ is of degree one, note first that by Remark 3.4, $G \otimes V$ is a reducing submodule of $G \otimes d \cdot E=d \cdot S$, and Proposition 9.1 implies that the closure of $\mathcal{E}_{V}$ is the space of all elements $\xi \in S=G \otimes E$ such that

$$
\left(Z_{1}^{*} \xi, \ldots, Z_{d}^{*} \xi\right) \in G \otimes V .
$$

Let $Q \in \mathcal{B}(d \cdot E)$ be the projection of $d \cdot E$ onto $V^{\perp}$. If we realize elements of $d \cdot E$ as column vectors with components in $E$, then the preceding formula makes the assertion

$$
\xi \in M^{\perp} \Longleftrightarrow(\mathbf{1} \otimes Q)\left(\begin{array}{c}
Z_{1}^{*} \xi \\
\vdots \\
Z_{d}^{*} \xi
\end{array}\right)=0 .
$$

We can realize $Q$ as a $d \times d$ matrix $\left(Q_{i j}\right)$ of operators in $\mathcal{B}(E)$. Letting $L_{i}$ be the operator in $\mathcal{B}(S)$ defined by

$$
L_{i}=Z_{1}^{*} \otimes Q_{i 1}+Z_{2}^{*} \otimes Q_{i 2}+\cdots+Z_{d}^{*} \otimes Q_{i d}, \quad i=1, \ldots, d,
$$

then (9.9) becomes the assertion

$$
M^{\perp}=\operatorname{ker} L_{1} \cap \cdots \cap \operatorname{ker} L_{d},
$$

or equivalently,

$$
M=\overline{\operatorname{ran} L_{1}^{*}+\cdots+\operatorname{ran} L_{d}^{*}}
$$

Finally, after noting that $Q_{i j}^{*}=Q_{j i}$ and each operator $L_{i}^{*}$ has the form

$$
L_{i}^{*}=Z_{1} \otimes Q_{1 i}+Z_{2} \otimes Q_{2 i}+\cdots+Z_{d} \otimes Q_{d i},
$$

it follows by inspection that the closure of ran $L_{i}^{*}$ is a graded degree-one submodule of $Z_{1} S+\cdots+Z_{d} S$. Hence these $d$ submodules generate a graded degree-one submodule $M \subseteq Z_{1} S+\cdots+Z_{d} S$.

Conversely, let $M$ be a degree-one submodule of $Z_{1} S+\cdots+Z_{d} S$, so that $M=$ $\left[\mathcal{A} M_{1}\right]$ where $M_{1}$ is a linear subspace of $S_{1}$. Consider the homomorphism of $\mathcal{A}$ modules $L: d \cdot S \rightarrow S$ defined by

$$
L\left(\eta_{1}, \ldots, \eta_{d}\right)=Z_{1} \eta_{1}+\cdots+Z_{d} \zeta_{d} .
$$

$L$ is a bounded linear map with closed range $Z_{1} S+\cdots+Z_{d} S$. By normalization of the basis $z_{1}, \ldots, z_{d}$ for $G_{1}$, it follows that for every $\xi \in M_{1} \subseteq G_{1} \otimes E$, the elements $\zeta_{k} \in E$ defined by $1 \otimes \zeta_{k}=Z_{k}^{*} \xi$ satisfy

$$
\xi=z_{1} \otimes \zeta_{1}+\cdots+z_{d} \otimes \zeta_{d}=L\left(\zeta_{1}, \ldots, \zeta_{d}\right) .
$$

Indeed, the set $W=\left\{\left(\zeta_{1}, \ldots, \zeta_{d}\right) \in d \cdot E: L\left(\zeta_{1}, \ldots, \zeta_{d}\right) \in M_{1}\right\}$ is a linear subspace of $d \cdot E$ such that $L(W)=M_{1}$. It follows that $N=[\mathcal{A} W]$ is a reducing submodule of $d \cdot S$ with the property that the restriction $L_{0}$ of $L$ to $N=[\mathcal{A} W]$ carries $N$ onto a dense linear submanifold of $M$. Note, however, that we cannot assert that $L(N)$ is closed.

In any case, we have

$$
M^{\perp}=L(N)^{\perp}=\operatorname{ker}\left(P_{N} L^{*}\right)=\left\{\xi \in S: L^{*} \xi \in N^{\perp}\right\} .
$$

After noting that $N^{\perp}=G \otimes W^{\perp}$ and that $L^{*}: S \rightarrow d \cdot S$ is the operator $L^{*} \xi=$ $\left(Z_{1}^{*} \xi, \ldots, Z_{d}^{*} \xi\right)$, we find that

$$
M^{\perp}=\left\{\xi \in S:\left(Z_{1}^{*} \xi, \ldots, Z_{d}^{*} \xi\right) \in G \otimes V\right\},
$$


where $V$ is the orthocomplement of $W$ in $d \cdot E$. Finally, since $M^{\perp}$ is the closure of the set of polynomials it contains, we have exhibited $M^{\perp}$ as the closure of the set of polynomials $f \in \mathcal{A} \otimes E$ with the property

$$
D_{G} f \in G \otimes V .
$$

Now by (9.5), $D_{G}$ has the form $D_{G}=u(N) \nabla$ where $u$ is a bounded function of the number operator, and where $u$ has the further property $u(k)>0$ for every $k=0,1,2, \ldots$. Since $G \otimes V$ is graded and graded submodules are invariant under functions of the number operator, it follows that

$$
M^{\perp}=\overline{\{f \in \mathcal{A} \otimes E: \nabla f \in \mathcal{A} \otimes V\}} .
$$

Proposition 9.1 provides a pointwise description of the relation $\nabla f \in \mathcal{A} \otimes V$ that exhibits $M^{\perp}$ as the closure of $\mathcal{E}_{V}$, as asserted in (9.8).

It is quite easy to adapt the proof of Theorem 9.3 so as to give a concrete description of $G(1)$-quotients for arbitrary graded completions $G$. The general statement is somewhat less elementary, in that one must replace the natural differentiation operators $\frac{\partial}{\partial z_{k}}$ with $Z_{k}^{*}, 1 \leq k \leq d$, and $\nabla$ with the operator $D_{G}$ of (9.2). Thus, in order to apply the more general result to a given graded completion $G$, one would first have to identify the operators $Z_{k}^{*}$ more explicitly as differential operators involving $\frac{\partial}{\partial z_{k}}$. We merely state the general result here, leaving the details for the reader.

Theorem 9.4. Let $S=G \otimes E$ be a standard Hilbert module based on an arbitrary graded completion $G$. The most general degree-one submodule of $Z_{1} S+\cdots+Z_{d} S$ has the form $M=\mathcal{E}_{V}^{\perp}$, where $V$ is a linear subspace of $d \cdot E, \mathcal{E}_{V}$ is defined by

$$
\mathcal{E}_{V}=\left\{f \in \mathcal{A} \otimes E: D_{G} f(z) \in V, \quad z \in \mathbb{C}^{d}\right\},
$$

and $D_{G}: \mathcal{A} \otimes E \rightarrow \mathcal{A} \otimes d \cdot E$ is the row operator $\left(Z_{1}^{*}, \ldots, Z_{d}^{*}\right)$. Thus the corresponding $G(1)$-quotient is identified with the closure of $\mathcal{E}_{V}$ in $G \otimes E$, whose operators are the compressions of $Z_{1}, \ldots, Z_{d}$ to $\overline{\mathcal{E}_{V}}$.

\section{Concluding REMARKs, OPEn PROBLEMS}

There is a range of conjectures associated with the basic problem discussed above. The most conservative of them is formulated below as Conjecture A. Since there is too little evidence to support the strongest conjecture one might entertain, we have formulated that as a question in Problem B.

Conjecture A. Let $G$ be a maximally symmetric essentially normal graded completion (such as the Hardy or Bergman modules of the unit ball or the space of the $d$-shift). Then for every finite-dimensional Hilbert space $E$ and every subspace $V \subseteq d \cdot E$, the $G(1)$-quotient $H_{V}=(G \otimes E) / \mathcal{E}_{V}^{\perp}$ described in Theorem 9.3 is essentially normal.

Problem B. Fix $p \in[1, \infty)$. Let $G$ be an arbitrary graded completion that is $p$-essentially normal, let $E$ be a finite-dimensional Hilbert space, and let $V$ be a subspace of $d \cdot E$. Is the $G(1)$-quotient $H_{V}=(G \otimes E) / \mathcal{E}_{V}^{\perp} p$-essentially normal?

By Corollary 8.4, an affirmative answer to Conjecture A would imply that all $G$-quotients are essentially normal in the maximally symmetric case. A positive reply to Problem B would have more far-reaching consequences. 
Remark 10.1 (Failure of essential normality). At this point we should point out that many familiar graded completions $G$ are not essentially normal; consequently one cannot expect quotients of standard Hilbert modules based on such $G$ to be essentially normal. For example, consider the Hardy or Bergman space $G$ of the bidisk $D \times D, D=\{z \in \mathbb{C}:|z|<1\}$. In either case, $G$ is a tensor product of one-dimensional Hilbert modules $G_{0} \otimes G_{0}$ where $G_{0}$ is the Hardy or Bergman space of the unit disk. Thus in both cases, the $C^{*}$-algebra generated by the coordinate multiplications of $G$ is the tensor product $\mathcal{T} \otimes \mathcal{T}$, where $\mathcal{T}$ is the one-dimensional Toeplitz $C^{*}$-algebra. Since $\mathcal{T} \otimes \mathcal{T}$ admits nontrivial chains of ideals such as

$$
\mathcal{K} \otimes \mathcal{K} \subseteq \mathcal{K} \otimes \mathcal{T} \subseteq \mathcal{K} \otimes \mathcal{T}+\mathcal{T} \otimes \mathcal{K} \subseteq \mathcal{T} \otimes \mathcal{T}
$$

$\mathcal{K}$ denoting the compact operators on $G_{0}, \mathcal{T} \otimes \mathcal{T}$ is not commutative modulo compact operators. See DH71, Dou05b for further discussion. The Bergman spaces of more general domains can give rise to type $I C^{*}$-algebras with arbitrarily long composition series Upm84.

There is considerable evidence to support Conjecture A and its consequences. The results of Arv04 imply that $H^{2}$-quotients $\left(H^{2} \otimes E\right) / M$ are $p$-essentially normal for $p>d$ whenever $M$ is generated by monomials. Douglas Dou05a has generalized that result to the context of more general weighted shifts. In dimension $d=2$, Guo Guo04 obtained trace estimates which imply that $H^{2} / M$ is essentially normal for every graded submodule $M \subseteq H^{2}$. In very recent work with Wang GW05, that result is improved; the new version implies that Conjecture $\mathrm{A}$ is true in dimension $d=2$.

Finally, we have shown that Conjecture A itself holds in certain special cases (in arbitrary dimensions), including a) that in which $V$ is one dimensional, b) that in which $V$ is of codimension 1 in $d \cdot E$, and c) that in which the submodule $M=\mathcal{E}_{V}^{\perp}$ is "diagonal" in the sense that $Z_{k}^{*} M_{1} \perp Z_{j}^{*} M_{1}$ when $k \neq j$. Unfortunately, none of the three proofs appears to generalize to the full context of Conjecture A.

\section{ACKNOWLEDGEMENT}

I want to thank Kunyu Guo for pointing out an error in a lemma of a previous draft of this paper: a condition that was asserted to hold for $n=1,2, \ldots$ actually holds only for $n=2,3, \ldots$. That opened a gap in the proof of the main result that remains unfilled at the time of this writing. The current version of this paper has been revised and reorganized in essential ways, and contains a more modest main result.

\section{Appendix A. EXAMPles With MAXIMUM SYMmetry}

We find all graded completions that are essentially normal and have maximum possible symmetry. This means that the associated inner product on $\mathcal{A}$ is invariant under the action of the full unitary group of $\mathbb{C}^{d}$ on $\mathcal{A}$.

Examples of maximally symmetric graded completions include the module $H^{2}$ of the $d$-shift, the Hardy module on the unit sphere in complex dimension $d$, the Bergman module on the ball, and many others related to domains with symmetry that are not tied to the unit sphere. Of course, there is a vast array of more general standard Hilbert modules that have less symmetry, even examples with minimum symmetry in the sense that only the center of the unitary group acts naturally, minimum symmetry being necessary as part of the definition of graded 
inner product. However, while the problem of classifying standard Hilbert modules in general appears difficult, we are optimistic about further progress in analyzing well-chosen intermediate subclasses.

The unitary group $G=\mathcal{U}_{d}$ of $\mathbb{C}^{d}$ acts naturally on the algebra $\mathcal{A}$ of polynomials in $d$ variables. One sees this most clearly by realizing $\mathcal{A}$ as the symmetric tensor algebra

$$
\mathbb{C} \dot{+} Z \dot{+} Z^{(2)} \dot{+} \cdots
$$

over the one-particle space $Z=\mathbb{C}^{d}, Z^{(n)}$ denoting the symmetric tensor product of $n$ copies of $Z$, which of course can be identified with the space $\mathcal{A}_{n} \subseteq \mathbb{C}\left[z_{1}, \ldots, z_{d}\right]$ of homogeneous polynomials of degree $n$ once one specifies a basis for $Z$. The action of $G$ is given by second quantization

$$
\Gamma(U)=\mathbf{1}_{\mathbb{C}} \dot{+} U \dot{+} U^{2} \dot{+} \cdots, \quad U \in G,
$$

where $U^{n}$ denotes the restriction of $U^{\otimes n} \in \mathcal{B}\left(Z^{\otimes n}\right)$ to the symmetric subspace $\mathcal{A}_{n} \subseteq Z^{\otimes n}, n=1,2, \ldots$

There are many graded completions in dimension $d$ that are associated with rotationally-invariant measures on $\mathbb{C}^{d}$. Indeed, let $\mu$ be a compactly supported probability measure on $\mathbb{C}^{d}$ that is invariant under the action of the full unitary group $\mathcal{U}_{d}$. The closed subspace $G \subseteq L^{2}(\mu)$ generated by the polynomials defines a Hilbert module which, under appropriate mild conditions on the measure $\mu$, is a $p$-essentially normal graded completion for every $p>d$ (see Theorem A.1 and Proposition A.3). The Hardy and Bergman modules in dimension $d$ are of this type.

These examples are obviously subnormal Hilbert modules. On the other hand, while the module $H^{2}$ of the $d$-shift is not subnormal and cannot be associated with a measure, it is also a symmetric graded completion Arv98.

Choose a $G$-invariant inner product $\langle\cdot, \cdot\rangle$ on $\mathcal{A}$. Such an inner product is of course graded, so that $\left\langle\mathcal{A}_{m}, \mathcal{A}_{n}\right\rangle=\{0\}$ if $m \neq n$. Moreover, since the restriction of $\Gamma$ to each homogeneous subspace $\mathcal{A}_{n}$ is an irreducible representation of $G$, any two $G$-invariant inner products on $\mathcal{A}_{n}$ must be proportional. Hence there is a sequence of positive constants $c_{0}, c_{1}, \ldots$ such that

$$
\langle f, g\rangle=c_{n}\langle f, g\rangle_{H^{2}}, \quad f, g \in \mathcal{A}_{n}, \quad n=0,1,2, \ldots,
$$

where $\langle\cdot, \cdot\rangle_{H^{2}}$ denotes the inner product of the symmetric Fock space. Conversely, given any sequence $c_{0}, c_{1}, \ldots$ of positive numbers, the preceding formula defines a $G$-invariant inner product $\langle\cdot, \cdot\rangle$ on $\mathcal{A}$. Thus, we seek to determine all sequences $c_{0}, c_{1}, c_{2}, \ldots$ with the property that the associated inner product leads to a standard Hilbert module that is essentially normal, or more generally, that is $p$-essentially normal for some $p>d$.

Fixing a sequence of positive numbers $c_{0}, c_{1}, \ldots$, it is more convenient to work with another sequence $\rho_{0}, \rho_{1}, \rho_{2}, \ldots$ defined by

$$
\rho_{k}=\sqrt{\frac{c_{k+1}}{c_{k}}}, \quad k=0,1,2, \ldots
$$

Thus, $c$ and $\rho$ are related by $c_{k+1}=\left(\rho_{0} \rho_{1} \cdots \rho_{k}\right)^{2} c_{0}, k=0,1,2, \ldots$, so that knowing the sequence $\rho_{0}, \rho_{1}, \cdots$ is equivalent to knowing the inner product up to a positive scaling factor.

Theorem A.1. Let $\rho_{0}, \rho_{1}, \ldots$ be a sequence of positive numbers, let $G$ be the Hilbert space obtained by completing $\mathcal{A}$ in the symmetric inner product associated with $\left(\rho_{k}\right)$ 
as above. Then the coordinate operators $Z_{1}, \ldots, Z_{d}$ are bounded and $Z_{1} G+\cdots+Z_{d} G$ is closed iff

$$
0<\epsilon \leq \rho_{k} \leq M<\infty, \quad k=0,1,2, \ldots,
$$

for some positive constants $\epsilon, M$. In that event, $G$ is also essentially normal iff the sequence $\rho_{n}$ oscillates slowly in the sense that

$$
\lim _{n \rightarrow \infty}\left(\rho_{n+1}-\rho_{n}\right)=0 .
$$

Proof. Let $\langle\cdot, \cdot\rangle$ be the inner product on $\mathcal{A}$ associated with

$$
c_{k}=\left(\rho_{0} \rho_{1} \cdots \rho_{k}\right)^{2} c_{0}, \quad k=0,1,2 \ldots,
$$

and let $\langle\cdot, \cdot\rangle_{H^{2}}$ be the inner product of the symmetric Fock space $H^{2}$. Let $\left(S_{1}, \ldots\right.$, $\left.S_{d}\right) \in \mathcal{B}\left(H^{2}\right)$ be the $d$-shift, let $E_{n} \in \mathcal{B}\left(H^{2}\right)$ be the projection onto the space of homogeneous polynomials $\mathcal{A}_{n}, n=0,1,2, \ldots$, and let $\Delta$ be the following diagonal operator in $\mathcal{B}\left(H^{2}\right)$ :

$$
\Delta=\sum_{n=0}^{\infty} \rho_{n} E_{n+1} .
$$

We claim first that, up to a graded unitary equivalence, the $d$-tuple $\left(Z_{1}, \ldots, Z_{d}\right)$ acting on $\mathcal{A} \subseteq G$ is the "weighted $d$-shift" $\left(\Delta S_{1}, \ldots, \Delta S_{d}\right)$, considered as a densely defined operator acting on $\mathcal{A} \subseteq H^{2}$. Indeed, we have

$$
\langle f, g\rangle=c_{n}\langle f, g\rangle_{H^{2}}, \quad f, g \in \mathcal{A}_{n}, \quad n=0,1,2, \cdots .
$$

Letting $W: \mathcal{A} \rightarrow \mathcal{A}$ be the linear map

$$
W=\sum_{n=0}^{\infty} \sqrt{c_{n}} E_{n},
$$

one sees that $W$ is a linear isomorphism of $\mathcal{A}$ onto itself, and by (A.4) we can take $G$ to be the completion of $\mathcal{A}$ in the inner product $f, g \mapsto\langle W f, W g\rangle_{H^{2}}$. For $S_{1}, \ldots, S_{d}$ and $Z_{1}, \ldots, Z_{d}$ as above we have $Z_{k} f=z_{k} \cdot f=S_{k} f$ for polynomials $f$; hence

$$
\left\langle Z_{k} f, g\right\rangle=\left\langle W S_{k} f, W g\right\rangle_{H^{2}}=\left\langle W S_{k} W^{-1} W f, W g\right\rangle_{H^{2}}
$$

and it follows that the $d$-tuple of restrictions of $Z_{1}, \ldots, Z_{d}$ to $\mathcal{A} \subseteq H$ is unitarily equivalent to the $d$-tuple of restrictions of $W S_{1} W^{-1}, \ldots, W S_{d} W^{-1}$ to $\mathcal{A} \subseteq H^{2}$. Using the commutation formula $S_{k} E_{n}=E_{n+1} S_{k}$, one can now compute in the obvious way to obtain $W S_{k} W^{-1}=\Delta S_{k}$, as asserted.

Thus we may take $Z_{k}=\Delta S_{k}, k=1, \ldots, d$. Since $S_{1} S_{1}^{*}+\cdots+S_{d} S_{d}^{*}$ is the projection $E_{0}^{\perp}=E_{1}+E_{2}+\cdots$, one finds that

$$
Z_{1} Z_{1}^{*}+\cdots+Z_{d} Z_{d}^{*}=\Delta\left(S_{1} S_{1}^{*}+\cdots+S_{d} S_{d}^{*}\right) \Delta=\Delta^{2}=\sum_{n=0}^{\infty} \rho_{n}^{2} E_{n+1},
$$

from which the equivalences characterized by (A.1) are apparent.

We now consider essential normality of the $Z_{k}=\Delta S_{k}, 1 \leq k \leq d$. Noting that the commutation formula $S_{k} E_{n}=E_{n+1} S_{k}$ implies that $\Delta$ commutes with $S_{k} S_{j}^{*}$, and that $\Delta S_{k}=S_{k} \tilde{\Delta}$ where

$$
\tilde{\Delta}=\sum_{n=0}^{\infty} \rho_{n} E_{n}
$$


it follows that each commutator $\left[Z_{j}^{*}, Z_{k}\right]$ can be written as

$$
S_{j}^{*} \Delta^{2} S_{k}-\Delta S_{k} S_{j}^{*} \Delta=S_{j}^{*} S_{k} \tilde{\Delta}^{2}-S_{k} S_{j}^{*} \Delta^{2}=\left[S_{j}^{*}, S_{k}\right] \tilde{\Delta}^{2}+S_{k} S_{j}^{*}\left(\tilde{\Delta}^{2}-\Delta^{2}\right) .
$$

Since the self-commutators $\left[S_{j}^{*}, S_{k}\right]$ are known to belong to $\mathcal{L}^{p}$ for every $p>d$ Arv98 and $\tilde{\Delta}^{2}$ is bounded, $\left[S_{j}^{*}, S_{k}\right] \tilde{\Delta}^{2}$ is compact. Hence $\left[Z_{j}^{*}, Z_{k}\right]$ is compact for all $j, k$ iff $S_{k} S_{j}^{*}\left(\tilde{\Delta}^{2}-\Delta^{2}\right)$ is compact for all $k, j$. Noting again that $S_{1} S_{1}^{*}+\cdots+S_{d} S_{d}^{*}$ is a rank-one perturbation of the identity, we find that $S_{k} S_{j}^{*}\left(\tilde{\Delta}^{2}-\Delta^{2}\right)$ is compact for all $j, k$ iff $\tilde{\Delta}^{2}-\Delta^{2}$ is compact. Since

$$
\tilde{\Delta}^{2}-\Delta^{2}=(\tilde{\Delta}+\Delta)(\tilde{\Delta}-\Delta)=(\tilde{\Delta}+\Delta)\left(\rho_{0} E_{0}+\sum_{n=1}^{\infty}\left(\rho_{n}-\rho_{n-1}\right) E_{n}\right)
$$

and $E_{0}, E_{1}, E_{2}, \ldots$ is a sequence of mutually orthogonal finite-dimensional projections, A.2 follows.

The examples characterized in Theorem A.1 include $H^{2}$ (take $\rho_{k}=1$ for every $k$ ), the Hardy module, which is associated with the sequence

$$
\rho_{k}=\sqrt{\frac{k+1}{k+d}}, \quad k=0,1,2, \ldots,
$$

and the Bergman module, which is associated with a similar sequence. While these examples are all tied directly to the unit sphere in the sense that $Z_{1} Z_{1}^{*}+\cdots+Z_{d} Z_{d}^{*}$ is a compact perturbation of the identity 1 , there are many others that are not.

For example, for any two positive constants $0<r_{1}<r_{2}<\infty$, the slowly oscillating sequence $\rho_{0}, \rho_{1}, \ldots$, defined by

$$
\rho_{k}^{2}=r_{1}+\left(r_{2}-r_{1}\right) \frac{1+\sin \sqrt{k}}{2}, \quad k=0,1,2, \ldots,
$$

defines an essentially normal standard Hilbert module with the property that the spectrum of $Z_{1} Z_{1}^{*}+\cdots+Z_{d} Z_{d}^{*}$ is the union $\{0\} \cup\left[r_{1}, r_{2}\right]$. Such a Hilbert module is associated with the annular region in $\mathbb{C}^{d}$,

$$
X=\left\{z \in \mathbb{C}^{d}: r_{1} \leq\|z\| \leq r_{2}\right\},
$$

since it gives rise to an exact sequence of $C^{*}$-algebras

$$
0 \longrightarrow \mathcal{K} \longrightarrow C^{*}\left(Z_{1}, \ldots, Z_{d}\right) \longrightarrow C(X) \longrightarrow 0 .
$$

Remark A.2 ( $p$-essential normality). Using the fact that the Hilbert module $H^{2}$ is $p$-essentially normal for every $p>d$ and that the dimension of $\mathcal{A}_{n}$ grows as a polynomial of degree $d-1$, it is not hard to adapt the proof of Theorem A.1 to establish the following characterization of $p$-essential normality:

Proposition A.3. Let $\rho_{0}, \rho_{1}, \ldots$ be a sequence satisfying (A.2) and property (ii) of Theorem A.1. Let $G$ be the graded completion obtained from the associated inner product. For every $p \in(d, \infty)$, the following are equivalent:

(i) $G$ is p-essentially normal.

$$
\sum_{k=1}^{\infty} k^{d-1}\left|\rho_{k+1}-\rho_{k}\right|^{p}<\infty
$$


We omit the proof since we do not require this result.

However, we point out that given some number $\alpha>d$, it is easy to use Proposition A.3 to find examples of sequences $\left(\rho_{k}\right)$ that give rise to graded completions $G$ that are $p$-essentially normal for all $p>\alpha>d$ but not for $p$ in the range $d<p \leq \alpha$. For instance, since the sequence of (A.6) behaves so that $\left|\rho_{k+1}-\rho_{k}\right|=O\left(k^{-1 / 2}\right)$, straightforward estimates using (A.7) show that the Hilbert module $S$ associated with that sequence is $p$-essentially normal iff $p>2 d$. In particular, this $S$ is not $p$-essentially normal for $p$ in the range $d<p \leq 2 d$.

\section{REFERENCES}

[Arv98] W. Arveson. Subalgebras of $C^{*}$-algebras III: Multivariable operator theory. Acta Math., 181:159-228, 1998. arXiv:funct-an/9705007. MR1668582 (2000e:47013)

[Arv00] W. Arveson. The curvature invariant of a Hilbert module over $\mathbb{C}\left[z_{1}, \ldots, z_{d}\right]$. J. Reine Angew. Math., 522:173-236, 2000. arXiv:math.OA/9808100. MR1758582(2003a:47013)

[Arv02] W. Arveson. The Dirac operator of a commuting $d$-tuple. Jour. Funct. Anal., 189:53-79, 2002. arXiv:math.OA/0005285. MR/1887629(2003a:47014)

[Arv04] W. Arveson. p-summable commutators in dimension d. J. Operator Theory, 2004. arXiv:math.OA/0308104 v2. MR/2168861

[BDF77] L.G. Brown, R. G. Douglas, and P. A. Fillmore. Extensions of $C^{*}$-algebras and $K$ Homology. Ann. of Math. (2), 105(2):265-324, March 1977. MR0458196 (56:16399)

[Cur81] R. Curto. Fredholm and invertible $n$-tuples of operators. The deformation problem. Trans. A.M.S., 266(1):129-159, 1981. MR613789 (82g:47010)

[DH71] R. G. Douglas and Roger Howe. On the $C^{*}$-algebra of Toeplitz operators on the quarterplane. Trans. A.M.S., 158:203-217, 1971. MR0288591 (44:5787)

[DM03] R. G. Douglas and G. Misra. Equivalence of quotient Hilbert modules. Proc. Indian Acad. Sci. (Math. Sci.), 113(3):281-291, August 2003. MR1999257 (2005g:46076)

[DMV00] R. G. Douglas, G. Misra, and C. Varughese. On quotient modules - the case of arbitrary multiplicity. J. Funct. Anal., 174:364-398, 2000. MR1768979 (2001f:47012)

[Dou05a] R. G. Douglas. Essentially reductive Hilbert modules. J. Operator Theory, 2005. arXiv:math.OA/0404167.

[Dou05b] R. G. Douglas. Ideals in Toeplitz algebras. Houston Math. J., 31:529-539, 2005. MR2132850 (2005m:46091)

[GS05] S. Gleason, J. Richter and C. Sundberg. On the index of invariant subspaces in spaces of analytic functions in several complex variables. to appear in Crelle's Journal, 2005.

[Guo04] K. Guo. Defect operators for submodules of $H_{d}^{2}$. J. Reine Angew. Math., 573:181-209, 2004. MR2084587 (2005h:47014)

[GW05] K. Guo and K. Wang. Essentially normal Hilbert modules and $K$-homology. preprint, 2005.

[MV93] V. Müller and F.-H. Vasilescu. Standard models for some commuting multioperators. Proc. Amer. Math. Soc., 117:979-989, 1993. MR1112498 (93e:47016)

[Tay70a] J. L. Taylor. The analytic functional calculus for several commuting operators. Acta Math, 125:1-38, 1970. MR 42 6622. MR0271741(42:6622)

[Tay70b] J. L. Taylor. A joint spectrum for several commuting operators. J. Funct. Anal., 6:172191, 1970. MR 42 3603. MR0268706 (42:3603)

[Upm84] Harald Upmeier. Toeplitz $C^{*}$-algebras on bounded symmetric domains. Ann. Math., 119(3):549-576, 1984. MR744863 (86a:47022)

Department of Mathematics, University of California, Berkeley, California 94720

E-mail address: arveson@math.berkeley.edu 